\title{
Semi-cooperative Strategies for Differential Games
}

\author{
Alberto Bressan and Wen Shen \\ S.I.S.S.A., Via Beirut 4, 34014 Trieste, Italy \\ Email: bressan@sissa.it, shen@sissa.it \\ and \\ Dept of Math, Penn State University, University Park, PA 16802, USA \\ Email: bressan@math.psu.edu, shen_w@math.psu.edu
}

April 19, 2004

\begin{abstract}
The paper is concerned with a non-cooperative differential game for two players. We first consider Nash equilibrium solutions in feedback form. In this case, we show that the Cauchy problem for the value functions is generically ill-posed. Looking at vanishing viscosity approximations, one can construct special solutions in the form of chattering controls, but these also appear to be unstable.

In the second part of the paper we propose an alternative "semi-cooperative" pair of strategies for the two players, seeking a Pareto optimum instead of a Nash equilibrium. In this case, we prove that the corresponding Hamiltonian system for the value functions is always weakly hyperbolic.
\end{abstract}

\section{Introduction}

A differential game models a situation where two or more individuals operate in a same environment, with different (possibly conflicting) goals. We shall assume here that the game has finite duration in time, and that the payoff of each player depends on the state of the system during the game or at the terminal time, and on the cost incurred while implementing his strategy.

For example, consider two companies which expect to launch on the market the same type of product, at a future time $T$. Let $x(t) \in[0,1]$ be the market share of the first company at time $t \in[0, T]$, while $1-x(t)$ is the market share of the second. Calling $u_{1}(t), u_{2}(t)$ the intensity of advertising by the two companies, the change in time of this market share may be described by the differential equation

$$
\frac{d}{d t} x(t)=x(1-x)\left[u_{1}(t)-u_{2}(t)\right] .
$$


The profits of two companies will depend on their share of the market at the time $T$, minus the cost of advertising, say

$$
J_{1} \doteq g_{1}(x(T))-\int_{0}^{T} h_{1}\left(u_{1}(t)\right) d t, \quad J_{2} \doteq g_{2}(1-x(T))-\int_{0}^{T} h_{2}\left(u_{2}(t)\right) d t,
$$

where $g_{1}, g_{2}, h_{1}, h_{2}$ are positive, increasing functions of their arguments. Notice that here the relevant state of the system, i.e. the market share, is described by the onedimensional variable $x=x(t)$.

In this context, the goal of a mathematical analysis is to indicate "optimal" or at least "rational" strategies for the various players, and predict actual behavior in real-life situations. A major step in the understanding of non-cooperative games with several players was provided by the concept of non-cooperative equilibrium, introduced by J. Nash [Na]. We recall that a set of strategies implemented by the various players constitutes a Nash equilibrium if, whenever one single player modifies his strategy, his own payoff will not increase.

This concept was first formulated in the context of static games, where no time-evolution is involved. It is natural to explore the relevance of Nash equilibria also in connection with differential games. We remark that in this case the information available to the players is a crucial aspect of the game. If each player has knowledge only of the initial state of the system, then his strategy must be a function of time only, say $u_{i}=U_{i}(t)$. Such strategies are called open-loop strategies. On the other hand, if the players can directly observe the state $x(t)$ of the system at every time $t \in[0, T]$, than they can adopt feedback strategies: $u_{i}=U_{i}(t, x)$ also depending on $x$. For the two cases of open-loop and feedback (closed-loop) strategies, the Nash equilibrium solutions can be substantially different.

Results on the existence of open-loop strategies can be found in [F1], [VZ]. In the present paper, we analyze the existence and stability of Nash equilibrium strategies in feedback form. Assuming that the dynamic of the system and the payoffs are given by smooth functions, the problem can be attacked using tools from P.D.E. theory. As in the standard theory of optimal control, the basic objects of our study are the value functions $V_{i}$. Roughly speaking, $V_{i}(\tau, y)$ denotes the payoff expected by the $i$-th player, if the game were to start at time $\tau$, with the system in the state $x(\tau)=y$. As shown in $[\mathrm{F} 1]$, these value functions satisfy a system of first order partial differential equations, with data specified at the terminal time $t=T$. In turn, from the gradients of these value functions one can recover the Nash feedback strategies for the various players.

The key question is whether this system of Hamilton-Jacobi equations for the value functions admits a solution. Moreover, is this solution unique? How is it affected by small perturbations of the data? Classical P.D.E. theory provides conditions under which the Cauchy problem is "well-posed", i.e. it admits a unique solution depending continuously on the initial data. The basic requirement is that the system should by hyperbolic. For a given system of P.D.E's, hyperbolicity amounts to an algebraic condition on the matrices of coefficients and could be checked in practice. 
We mention that the special case of two-players, zero-sum games has been widely studied in the literature. In this case, the value functions satisfy $V_{1}(t, x)=-V_{2}(t, x)$. Hence the system of P.D.E's reduces to a single scalar Hamilton-Jacobi equation for $V_{1}$, which is now well understood thanks to the theory of viscosity solution of Crandall and Lions, see $[\mathrm{BC}]$.

In [BS] we studied differential games where the state of the system is described by a one-dimensional variable, and derived sufficient conditions for the corresponding system to be hyperbolic. In the positive case, we proved that the Cauchy problem is well-posed, whose solution would in turn yield a Nash equilibrium solution.

In the present paper we show that, when the state space is multi-dimensional, the corresponding system of P.D.E's is generically not hyperbolic, and the Cauchy problem is not well-posed. We study in detail a particular one-dimensional example where hyperbolicity fails, and construct a family of unstable, highly oscillatory solutions. Our conclusion is that the concept of Nash equilibrium is not appropriate for the study of feedback strategies for differential games in continuous time. Indeed, solutions are extremely sensitive to small perturbations of the data, so that the mathematical model has no predictive power.

To readdress the situation, one possibility is to introduce some stochasticity in the system [F2, Mn]. The presence of random inputs, in the form of white noise, has a well known stabilizing effect since it transforms the system into a parabolic one. Another possibility, explored in the present paper, is to allow some degree of cooperation among the players. As proved by Smale in connection with the repeated prisoner's dilemma [Sm], even if the players do not communicate with each other, over a period of time they can devise strategies converging to a Pareto optimum. In the setting of differential games we prove that, if these semi-cooperative strategies are implemented, then the system of P.D.E's for the value functions turns out to be always hyperbolic, at least in a weak sense. Partial cooperation thus removes the most severe instabilities found among Nash non-cooperative equilibrium solutions.

\section{Outline of the paper}

In this paper we study feedback solutions for a non-cooperative differential game involving two players. The evolution of the system is governed by a differential equation of the form

$$
\dot{x}(t)=f_{1}\left(x, u_{1}\right)+f_{2}\left(x, u_{2}\right),
$$

with initial data

$$
x(\tau)=y \in \mathbb{R}^{m} .
$$

Here $t \mapsto u_{i}(t) \in \Lambda_{i}$ is the control implemented by the $i$-th player $(i=1,2)$. We assume that each $\Lambda_{i}$ is a subset of some Euclidean space $\mathbb{R}^{n}$. Together with (2.1) we consider 
the payoff functionals

$$
J_{i}=J_{i}\left(\tau, y, u_{1}, u_{2}\right) \doteq g_{i}(x(T))-\int_{\tau}^{T} h_{i}\left(x(t), u_{i}(t)\right) d t,
$$

consisting of a terminal payoff $g_{i}$ and a running cost $h_{i}$. The goal of the $i$-th player is to maximize $J_{i}$. We recall that a pair of feedback strategies

$$
u_{1}=U_{1}^{*}(t, x), \quad u_{2}=U_{2}^{*}(t, x),
$$

is called a Nash equilibrium solution if the following holds. For each $i=1,2$, if the $i$-th player chooses an alternative strategy $U_{i}$ while the other player sticks to his previous strategy $U_{j}^{*}$, then the payoff for the $i$-th player does not increase. Namely

$$
J_{1}\left(\tau, y, U_{1}, U_{2}^{*}\right) \leq J_{i}\left(\tau, y, U_{1}^{*}, U_{2}^{*}\right), \quad J_{2}\left(\tau, y, U_{1}^{*}, U_{2}\right) \leq J_{i}\left(\tau, y, U_{1}^{*}, U_{2}^{*}\right),
$$

for every alternative strategies $U_{1}, U_{2}$. Assume that a value function $V=\left(V_{1}, V_{2}\right)$ exists, so that $V_{i}(t, x)$ is the payoff for the $i$-th player, when the game starts from the position $x$ at time $t$ and everyone plays optimally but no cooperation is allowed. Under suitable regularity conditions (see [F1], p.292), the function $V$ provides a solution to the system of Hamiltonian equations

$$
\frac{\partial}{\partial t} V_{i}+H_{i}\left(x, \nabla V_{1}, \nabla V_{2}\right)=0
$$

with terminal data

$$
V_{i}(T, x)=g_{i}(x) .
$$

The Hamiltonian functions $H_{i}$ are defined as follows. Assume that, for any given gradient vectors $p_{1}, p_{2} \in \mathbb{R}^{n}$, there exist optimal control values $u_{j}^{*}\left(x, p_{j}\right), j=1,2$, such that

$$
p_{j} \cdot f_{i}\left(x, u_{j}^{*}\left(x, p_{j}\right)\right)-h_{j}\left(x, u^{*}\left(x, p_{j}\right)\right)=\max _{\omega \in \Lambda_{j}}\left\{p_{j} \cdot f_{j}(x, \omega)-h_{j}(x, \omega)\right\} .
$$

Then

$$
H_{i}\left(x, p_{1}, p_{2}\right)=p_{i} \cdot\left[f_{1}\left(x, u_{1}^{*}\left(x, p_{1}\right)\right)+f_{2}\left(x, u_{2}^{*}\left(x, p_{2}\right)\right)\right]-h_{i}\left(x, u_{i}^{*}\left(x, p_{i}\right)\right) .
$$

In the one dimensional case, differentiating (2.5) w.r.t. $x$ one obtains a system of conservation laws for the gradient functions $p_{i} \doteq V_{i, x}$, namely

$$
p_{i, t}+H_{i}(x, p)_{x}=0 .
$$

In the earlier paper [BS], the authors derived conditions which guarantee that the system (2.9) is hyperbolic. If this happens, it was proved that the weak solution to this system of conservation laws yields a Nash equilibrium solution to the differential game.

The present paper is mainly concerned with the alternative case, where the system is not hyperbolic. In Section 3 we first observe that hyperbolicity generically fails for systems 
of the form (2.5), whenever the state $x$ lies in a space of dimension $n \geq 2$. This has severe implications for the existence of a Nash equilibrium feedback, because the Cauchy problem (2.5)-(2.6) is then ill-posed. Notice that the present situation is quite different from the case of two-person, zero-sum differential games, where the value function can be characterized as the unique viscosity solution of a scalar Hamilton-Jacobi equation (see $[\mathrm{BC}])$.

In Section 4 we focus our attention on a particular one-dimensional system, in a region where hyperbolicity fails. In an attempt to recover some stability, we first add a small viscosity and obtain the parabolic system

$$
\left\{\begin{array}{l}
p_{1, t}-\left(p_{1}^{2} / 2+p_{1} p_{2}\right)_{x}=\varepsilon p_{1, x x} \\
p_{2, t}-\left(p_{2}^{2} / 2+p_{1} p_{2}\right)_{x}=\varepsilon p_{2, x x}
\end{array}\right.
$$

Here the main interest is in the region where $p_{1} p_{2}<0$. By Fourier analysis, one easily checks that the constant solutions

$$
p_{1}(t, x)=\bar{p}_{1}, \quad p_{2}(t, x)=\bar{p}_{2}
$$

are unstable w.r.t. low frequency perturbations. We shall prove the existence of solutions of (2.10) in the form of periodic travelling waves. From the computations in the Appendix, it also follows the existence of special solutions which initially lie arbitrarily close to a constant state, but converge to a periodic travelling wave for $t \rightarrow \infty$. Letting the viscosity coefficient $\varepsilon \rightarrow 0+$, a periodic travelling wave of (2.10) yields a measurevalued solution of the corresponding system without viscosity (see [FL] for a similar approach to conservation laws inside an elliptic region). From the point of view of differential games, this limit represents a pair of Nash equilibrium strategies of chattering nature. The two players implement periodic controls $u_{i}(t)$ which oscillate with higher and higher frequency as $\varepsilon \rightarrow 0$. A further analysis, however, shows that even these periodic travelling wave solutions are unstable. More general instability results can be found in [OZ1, OZ2, S2]. In regions of ellipticity, the behavior of vanishing viscosity solutions thus appears to be extremely irregular. The eventual conclusion of our analysis is that, except for a few special cases, the concept of Nash non-cooperative equilibrium is not appropriate to study differential games in continuous time. Indeed, it generically leads to ill-posed Cauchy problems. The highly unstable nature of the solutions does not allow one to extract any useful information from the mathematical model.

In an attempt to readdress this situation, in the second part of the paper we propose some alternative strategies for the two players. These will be different from the Nash equilibrium strategies, and generally lead to well-posed Cauchy problems for the value functions.

If full cooperation were possible, then the players would simply choose the strategy that maximizes the sum $J_{1}+J_{2}$ of the two payoffs. In this case, $u_{1}, u_{2}$ can be regarded as components of one single control function. The optimization problem thus fits entirely within the framework of optimal control theory. The only additional feature arising 
from the differential game is the possible side payment that one of the players should make to the other, to preserve some fairness.

In the case where the two players cannot communicate and are not allowed to make side payments, their behavior can still drift away from a Nash equilibrium and approach a Pareto optimum which improves both of their payoffs. In connection with the iterated prisoner's dilemma, a mechanism which allows this transition was proposed by S. Smale [Sm]. In Section 5 we observe that these same ideas are appropriate also in continuous time. We call these strategies "semi-cooperative". At each given time, they seek the unique Pareto optimum which yields to both players the same improvement, compared with the Nash equilibrium.

When these semi-cooperative strategies are implemented, the equations (2.5) for the value functions become different. Indeed, the Hamiltonian functions $H_{i}\left(x, p_{1}, p_{2}\right)$ in (2.8) are now defined in terms of controls $u_{i}^{*}\left(x, p_{1}, p_{2}\right)$ attaining a Pareto optimum instead of a Nash equilibrium. A remarkable fact, proved in Section 6 , is that with these new Hamiltonians the system (2.5) is always weakly hyperbolic. This looks promising in connection with the Cauchy problem (2.5)-(2.6) for the value functions. Indeed, our semi-cooperative solutions will not experience the severe instabilities of the Nash solutions. It is thus expected that some existence theorem should hold in greater generality.

Remark 1. Throughout this paper we always consider a system with smooth, strictly convex running cost functions. This allows us to use basic P.D.E. theory in the analysis of the value function. On the other hand, earlier studies of differential games [CP], $[\mathrm{O}]$ were concerned with "degenerate" cases, where the running cost is piecewise affine, possibly taking the value $+\infty$. In these cases, a Nash equilibrium solution could be constructed "by hand", piecing together a finite number of trajectories (corresponding to constant controls), and instabilities would not be easily detected.

Another class of non-cooperative games that have received considerable attention are those with linear dynamics and quadratic running cost functions, as in [BO]. In this special case, at each time $t$ the value functions are always quadratic polynomials w.r.t. the space variables. The backward Cauchy problem corresponding to (2.5)-(2.6) thus reduces to a finite dimensional system of O.D.E's, which always has at least a local solution. This provides examples of possibly ill-posed Cauchy problems, where solutions are always found, thanks to the very special form of the data (quadratic polynomials).

\section{$3 \quad$ Generic instability}

We begin by recalling some basic results concerning systems of P.D.E's in several space dimensions [S1]. Consider the linear system on $\mathbb{R}^{m}$ with constant coefficients:

$$
\frac{\partial}{\partial t} v+\sum_{\alpha=1}^{m} A_{\alpha} \frac{\partial}{\partial x_{\alpha}} v=0 \text {. }
$$


Here $t$ is time, $x=\left(x_{1}, \ldots, x_{m}\right)$ is the space variable while $v=\left(v_{1}, \ldots, v_{n}\right)$ is the dependent variable. For each vector $\xi=\left(\xi_{1}, \ldots, \xi_{m}\right) \in \mathbb{R}^{m}$ we define the linear combination

$$
A(\xi) \doteq \sum_{\alpha} \xi_{\alpha} A_{\alpha} .
$$

Definition 1. The system (3.1) is hyperbolic if there exists a constant $C$ such that

$$
\sup _{\xi \in \mathbb{R}^{m}}\|\exp i A(\xi)\| \leq C
$$

Of course, the matrix exponential is defined as

$$
\exp i A(\xi) \doteq \sum_{k=0}^{\infty} \frac{(i A(\xi))^{k}}{k !} .
$$

The above definition is motivated by the following well known result [S1].

Lemma 1 The initial value problem for the system (3.1) is well-posed in $\mathbf{L}^{2}\left(\mathbb{R}^{m}\right)$ if and only if the system is hyperbolic.

Indeed, given an initial condition $u(0, x)$, the solution can be computed by Fourier transform. The vector valued function

$$
\hat{u}(t, \xi)=(2 \pi)^{-m / 2} \int_{\mathbb{R}^{m}} e^{-i \xi \cdot x} u(t, x) d x
$$

satisfies the evolution equation

$$
\frac{\partial}{\partial t} \hat{u}(t, \xi)=-i A(\xi) \hat{u}(t, \xi)
$$

Therefore

$$
\begin{gathered}
\hat{u}(t, \xi)=\exp (-i t A(\xi)) \hat{u}(0, \xi), \\
u(t, x)=(2 \pi)^{-m / 2} \int_{\mathbb{R}^{m}} e^{i \xi \cdot x} \hat{u}(t, \xi) d x .
\end{gathered}
$$

Observing that $t A(\xi)=A(t \xi)$, since the Fourier transform is an isometry on $\mathbf{L}^{2}$, we have

$$
\|u(t)\|_{\mathbf{L}^{2}}=\|\hat{u}(t)\|_{\mathbf{L}^{2}} \leq \sup _{\xi \in \mathbb{R}^{m}}\|\exp (-i t A(\xi))\| \cdot\|\hat{u}(0)\|_{\mathbf{L}^{2}}=\sup _{\xi \in \mathbb{R}^{m}}\|\exp i A(\xi)\| \cdot\|u(0)\|_{\mathbf{L}^{2}} .
$$

If (3.2) holds, then for any given time $t>0$, the flow map $u(0) \mapsto u(t)$ is a bounded linear operator on $\mathbf{L}^{2}\left(\mathbb{R}^{m}\right)$. Viceversa, if (3.2) fails, for any time $t>0$ and any constant $M$ we can find an open set of $\Omega \subset \mathbb{R}^{m}$ such that

$$
\|\exp (-i t A(\xi))\|>M
$$


for all $\xi \in \Omega$. Choosing a suitable initial data $u(0)$ whose Fourier transform $\hat{u}(0)$ is supported inside the set $\Omega$, one can thus construct a solution such that

$$
\begin{aligned}
\|u(t)\|_{\mathbf{L}^{2}} & =\|\hat{u}(t)\|_{\mathbf{L}^{2}}=\left(\int\|\exp (-i t A(\xi)) \hat{u}(0, \xi)\|^{2} d \xi\right)^{1 / 2} \\
> & \left(\int|M \hat{u}(0, \xi)|^{2} d \xi\right)^{1 / 2}=M\|\hat{u}(0)\|_{\mathbf{L}^{2}}=M\|u(0)\|_{\mathbf{L}^{2}} .
\end{aligned}
$$

Since $M$ can be arbitrarily large, we conclude that the solution operator is not continuous as a linear map within the space $\mathbf{L}^{2}\left(\mathbb{R}^{m}\right)$.

For an extension of this result to the case of linear systems with variable coefficients, see [Lx]. A necessary condition for hyperbolicity which can be easily checked in practice is the following.

Lemma 2 If the system (3.1) is hyperbolic, then for every $\xi \in \mathbb{R}^{m}$ the matrix $A(\xi)$ has $n$ real eigenvalues $\lambda_{1}(\xi), \ldots, \lambda_{n}(\xi)$.

Indeed, call $\rho(M)$ the spectral radius of a matrix $M$. The assumption of hyperbolicity implies

$$
\sup _{\xi \in \mathbb{R}^{m}} \rho(\exp i A(\xi))<\infty .
$$

Observing that, for every integer $k$,

$$
\exp i A(k \xi)=(\exp i A(\xi))^{k},
$$

we deduce that the spectral radii of the matrices

$$
\exp i A(\xi), \quad \exp (-i A(\xi))=(\exp i A(\xi))^{-1}
$$

are both $=1$. Since the eigenvalues of $\exp M$ are the exponentials of the eigenvalues of the matrix $M$, we conclude that all eigenvalues of $i A(\xi)$ must be purely imaginary. Hence all eigenvalues of $A(\xi)$ must be real.

The above lemma motivates

Definition 2. The system (3.1) is weakly hyperbolic if, for every $\xi \in \mathbb{R}^{m}$, all eigenvalues of the matrix $A(\xi)$ are real.

Notice that, by Lemma 2, every linear hyperbolic system is weakly hyperbolic. The viceversa does not hold. For example, in one space dimension the system $u_{t}+A u_{x}=0$ is hyperbolic if and only if the matrix $A$ admits a basis of real eigenvectors. This 
condition is strictly stronger than having real eigenvalues. According to the previous lemmas, weak hyperbolicity is thus a necessary (but not sufficient) condition for the Cauchy problem to be well-posed.

Next, consider a nonlinear system. A definition of hyperbolicity can still be given, by looking at the corresponding linearizations. To fix the ideas, consider the system of Hamilton-Jacobi equations in $m$ space dimensions

$$
\partial_{t} V_{i}+H_{i}\left(x, \nabla_{x} V_{1}, \ldots, \nabla_{x} V_{n}\right)=0 \quad i=1, \ldots, n .
$$

At a given point $\left(x, p_{1}, \ldots, p_{n}\right) \in \mathbb{R}^{(1+n) m}$, with

$$
x \in \mathbb{R}^{m}, \quad p_{i} \doteq \nabla_{x} V_{i}=\left(p_{i 1}, \ldots, p_{i m}\right) \in \mathbb{R}^{m},
$$

the linearization of (3.3) takes the form

$$
\frac{\partial v_{i}}{\partial t}+\sum_{j, \alpha}\left[\frac{\partial H_{i}}{\partial p_{j \alpha}}\left(x, p_{1}, \ldots, p_{n}\right)\right] \cdot \frac{\partial v_{j}}{\partial x_{\alpha}}=0 \quad i=1, \ldots, n .
$$

Observe that this is a linear system with constant coefficients of the form (3.1), with

$$
\left(A_{\alpha}\right)_{i j}=\frac{\partial H_{i}}{\partial p_{j \alpha}}\left(x, p_{1}, \ldots, p_{n}\right)
$$

Definition 3. The system (3.3) is hyperbolic (or weakly hyperbolic) on a domain $\Omega \subseteq \mathbb{R}^{(1+n) m}$ if, for every $\left(x, p_{1}, \ldots, p_{n}\right) \in \Omega$ the linearized system (3.4) is hyperbolic (weakly hyperbolic, respectively).

To study the stability of a solution to a nonlinear system, a key step is the analysis of the corresponding linearized system. In view of Lemmas 1 and 2, and their extension [Lx] to systems with non-constant coefficients, if weak hyperbolicity fails, small perturbations can be instantly amplified by an arbitrarily large factor. Hence the Cauchy problem cannot be well-posed.

In the remainder of this section we consider a system of Hamilton-Jacobi equations for the value functions in a noncooperative differential game, obtained as a Nash equilibrium. Our following two examples show that, in several space dimensions, in general this system is never weakly hyperbolic. Hence the Cauchy problem is not well-posed.

Example 1. Consider a game for two players in $m$ space dimensions, with dynamics

$$
\dot{x}=f_{0}(x)+u_{1}+u_{2}, \quad u_{1}, u_{2}:[0, T] \mapsto \mathbb{R}^{m} .
$$

As remarked in Section 6 of [BS], a larger class of games can be reduced to the form (3.6), by a suitable reparametrization. We consider the same initial data and payoff functionals 
as in (2.2)-(2.3). We assume that every function $h_{i}$ is uniformly convex w.r.t. $u_{i}$, so that the Hessian $D^{2} h_{i}$ is strictly positive definite at every point. Call $p_{i} \doteq \nabla_{x} V_{i}$ the gradient of the $i$-th value function $V_{i}$. The Nash equilibrium strategies are obtained as

$$
u_{i}^{*}\left(x, p_{i}\right)=\arg \max _{\omega}\left\{p_{i} \cdot \omega-h_{i}(x, \omega)\right\} .
$$

Note that, in the above expression, $\omega$ is allowed to range over the whole space $\mathbb{R}^{m}$. Since the gradient vanishes at a point of maximum, the above implies

$$
p_{i}-\nabla h_{i}\left(x, u_{i}^{*}\left(x, p_{i}\right)\right)=0 .
$$

The system of Hamilton-Jacobi equations for the value functions takes the form

$$
\left\{\begin{array}{l}
V_{1, t}+p_{1} \cdot\left[f_{0}(x)+u_{1}^{*}\left(x, p_{1}\right)+u_{2}^{*}\left(x, p_{2}\right)\right]-h_{1}\left(x, u_{1}^{*}\left(x, p_{1}\right)\right)=0 \\
V_{2, t}+p_{2} \cdot\left[f_{0}(x)+u_{1}^{*}\left(x, p_{1}\right)+u_{2}^{*}\left(x, p_{2}\right)\right]-h_{2}\left(x, u_{2}^{*}\left(x, p_{2}\right)\right)=0
\end{array}\right.
$$

In order to check hyperbolicity, we need to compute the eigenvalues of the matrix $A(\xi)$, for any unit vector $\xi \in \mathbb{R}^{m}$. For notational simplicity, we consider here the case $\xi=\mathbf{e}_{1}$, where $\mathbf{e}_{1}$ is the first vector in an orthonormal basis. One finds

$$
A_{1}=A\left(\mathbf{e}_{1}\right)=\left(\begin{array}{ll}
\frac{\partial H_{1}}{\partial p_{11}} & \frac{\partial H_{1}}{\partial p_{21}} \\
\frac{\partial H_{2}}{\partial p_{11}} & \frac{\partial H_{2}}{\partial p_{21}}
\end{array}\right) .
$$

Writing $u_{i}^{*}=\left(u_{i 1}^{*}, \cdots, u_{i m}^{*}\right) \in \mathbb{R}^{m}$ and $f_{0}=\left(f_{01}, \cdots, f_{0 m}\right) \in \mathbb{R}^{m}$, using (3.7) we obtain

$$
A\left(\mathbf{e}_{1}\right)=\left(\begin{array}{cc}
f_{01}+u_{11}^{*}+u_{21}^{*} & p_{1} \cdot D u_{2}^{*} \cdot \mathbf{e}_{1} \\
p_{2} \cdot D u_{1}^{*} \cdot \mathbf{e}_{1} & f_{01}+u_{11}^{*}+u_{21}^{*}
\end{array}\right) .
$$

Here $D u_{i}^{*}$ denotes the Jacobian matrix of first order derivatives of $u_{i}^{*}$ w.r.t. $p_{i}$. The matrix $A\left(\mathbf{e}_{1}\right)$ will have complex eigenvalues if and only if

$$
\left(p_{1} \cdot D u_{2}^{*} \cdot \mathbf{e}_{1}\right) \cdot\left(p_{2} \cdot D u_{1}^{*} \cdot \mathbf{e}_{1}\right)<0
$$

We now observe that, up to a rotation of the standard basis, any unit vector can be taken as $\mathbf{e}_{1}$. In particular, under the generic assumption that the two vectors $p_{1} \cdot D u_{2}^{*}$ and $p_{2} \cdot D u_{1}^{*}$ are not parallel, one can find a unit vector $\mathbf{e}_{1}$ such that

$$
p_{1} \cdot D u_{2}^{*} \cdot \mathbf{e}_{1}<0, \quad p_{2} \cdot D u_{1}^{*} \cdot \mathbf{e}_{1}>0 .
$$

Therefore, the system (3.6) is not weakly hyperbolic. 
Example 2. Let $f_{0}, f_{1}, f_{2}$ be smooth vector fields on $\mathbb{R}^{m}$ and consider a game for two players with dynamics

$$
\dot{x}=f_{0}(x)+f_{1}(x) u_{1}+f_{2}(x) u_{2}, \quad u_{1}, u_{2}:[0, T] \mapsto \mathbb{R} .
$$

The initial data and the payoff functionals are as in (2.2)-(2.3). In this case, the optimal controls are found to be

$$
u_{i}^{*}\left(x, p_{i}\right) \doteq \arg \max _{\omega}\left\{p_{i} \cdot f_{i}(x) \omega-h_{i}(x, \omega)\right\} .
$$

The value functions $V_{i}$ now satisfy the system of Hamilton-Jacobi equations

$$
\left\{\begin{array}{l}
V_{1, t}+p_{1} \cdot\left[f_{0}(x)+f_{1}(x) u_{1}^{*}\left(x, p_{1}\right)+f_{2}(x) u_{2}^{*}\left(x, p_{2}\right)\right]-h_{1}\left(x, u_{1}^{*}\left(x, p_{1}\right)\right)=0 \\
V_{2, t}+p_{2} \cdot\left[f_{0}(x)+f_{1}(x) u_{1}^{*}\left(x, p_{1}\right)+f_{2}(x) u_{2}^{*}\left(x, p_{2}\right)\right]-h_{2}\left(x, u_{2}^{*}\left(x, p_{2}\right)\right)=0 .
\end{array}\right.
$$

Notice that $u_{1}^{*}, u_{2}^{*}$ are now scalar functions. In connection with the unit vector $\mathbf{e}_{1}$, we find

$$
A_{1}=A\left(\mathbf{e}_{1}\right)=\left(\begin{array}{cc}
\left(f_{0}+f_{1} u_{1}^{*}+f_{2} u_{2}^{*}\right) \cdot \mathbf{e}_{1} & \left(p_{1} \cdot f_{2}\right)\left(\nabla u_{2}^{*} \cdot \mathbf{e}_{1}\right) \\
\left(p_{2} \cdot f_{1}\right)\left(\nabla u_{1}^{*} \cdot \mathbf{e}_{1}\right) & \left(f_{0}+f_{1} u_{1}^{*}+f_{2} u_{2}^{*}\right) \cdot \mathbf{e}_{1}
\end{array}\right)
$$

This matrix has complex eigenvalues if and only if

$$
\left(p_{1} \cdot f_{2}\right)\left(\nabla u_{2}^{*} \cdot \mathbf{e}_{1}\right) \cdot\left(p_{2} \cdot f_{1}\right)\left(\nabla u_{1}^{*} \cdot \mathbf{e}_{1}\right)<0 .
$$

Under generic conditions, the two inner products $\left(p_{1} \cdot f_{2}\right)$ and $\left(p_{2} \cdot f_{1}\right)$ are both $\neq 0$. Moreover the two gradients $\nabla u_{1}^{*}, \nabla u_{2}^{*}$ are not parallel. It is thus possible to find a unit vector $\mathbf{e}_{1}$ such that

$$
\left.\operatorname{sign}\left[\left(\nabla u_{1}^{*} \cdot \mathbf{e}_{1}\right)\left(\nabla u_{2}^{*} \cdot \mathbf{e}_{1}\right)\right)\right] \neq \operatorname{sign}\left[\left(p_{1} \cdot f_{2}\right)\left(p_{2} \cdot f_{1}\right)\right]
$$

Again we conclude that the hyperbolicity condition generically fails.

\section{A study of vanishing viscosity limits}

In this section we study in greater detail an example of a differential game on a domain where hyperbolicity fails. Consider a two-persons non-cooperative differential game in one space dimension, with the simple dynamics

$$
\dot{x}=u_{1}+u_{2}, \quad x(\tau)=y,
$$

and payoff functionals

$$
J_{i}=J_{i}\left(\tau, y, u_{1}, u_{2}\right)=g_{i}(x(T))-\int_{\tau}^{T} \frac{u_{i}^{2}}{2} d t \quad i=1,2 .
$$


Here $u_{i}$ is the control implemented by the $i$-th player, while $g_{i}$ is his terminal payoff. Let $V_{1}, V_{2}$ be the corresponding value functions, and call $p_{1} \doteq V_{1, x}$ and $p_{2} \doteq V_{2, x}$ their spatial derivatives. The corresponding optimal feedback control $u_{i}^{*}$ for the $i$-th player is

$$
u_{i}^{*}\left(p_{i}\right)=\arg \max _{\omega}\left\{p_{i} \cdot \omega-\left(\omega^{2} / 2\right)\right\}=p_{i},
$$

and the Hamiltonian functions are

$$
H_{i}\left(p_{1}, p_{2}\right)=\left(p_{1}+p_{2}\right) p_{i}-p_{i}^{2} / 2, \quad i=1,2 .
$$

Therefore $p=\left(p_{1}, p_{2}\right)$ satisfies a $2 \times 2$ system of conservation laws, solved backward in time

$$
p_{i, t}+H_{i}\left(p_{1}, p_{2}\right)_{x}=0, \quad p_{i}(x, T)=g_{i, x}(x) .
$$

Setting $\tau=T-t$, and still using $t$ as time variable, we obtain a more standard Cauchy problem, to be solved forward in time:

$$
\left\{\begin{array}{l}
p_{1, t}-\left(p_{1}^{2} / 2+p_{1} p_{2}\right)_{x}=0 \\
p_{2, t}-\left(p_{2}^{2} / 2+p_{1} p_{2}\right)_{x}=0
\end{array}\right.
$$

with the initial data

$$
p_{1}(0, x)=g_{1, x}(x), \quad p_{2}(0, x)=g_{2, x}(x) .
$$

The system (4.4) can be written in quasi-linear form

$$
p_{t}-A(p) p_{x}=0, \quad A(p) \doteq\left(\begin{array}{cc}
p_{1}+p_{2} & p_{1} \\
p_{2} & p_{1}+p_{2}
\end{array}\right) .
$$

The eigenvalues of the matrix $A(p)$ are real if $p_{1} p_{2} \geq 0$, and complex if $p_{1} p_{2}<0$. In particular, if the initial data are such that

$$
g_{1, x}(x)>0, \quad g_{2, x}(x)>0
$$

for all $x \in \mathbb{R}$, then by the analysis in [BS] a Nash equilibrium feedback solution can be obtained by solving the hyperbolic system of conservation laws (4.4). Throughout the following, we instead focus our attention on solutions with $p_{1} p_{2}<0$. As a first step, we add a small viscosity and consider the parabolic system

$$
p_{t}^{\varepsilon}-A\left(p^{\varepsilon}\right) p_{x}^{\varepsilon}=\varepsilon p_{x x}^{\varepsilon} .
$$

This system is related to a stochastic differential game with dynamics

$$
d x=\left(u_{1}+u_{2}\right) d t+\varepsilon d \omega,
$$

where $\omega$ denotes a standard Brownian motion, as in [F2]. Observe that $p^{\varepsilon}=\left(p_{1}^{\varepsilon}, p_{2}^{\varepsilon}\right)$ provides a solution to (4.7) if and only if

$$
p^{\varepsilon}(t, x)=p(t / \varepsilon, x / \varepsilon),
$$


where $p=\left(p_{1}, p_{2}\right)$ solves the system with unit viscosity

$$
\left\{\begin{array}{l}
p_{1, t}-\left(p_{1}^{2} / 2+p_{1} p_{2}\right)_{x}=p_{1, x x} \\
p_{2, t}-\left(p_{2}^{2} / 2+p_{1} p_{2}\right)_{x}=p_{2, x x} .
\end{array}\right.
$$

To achieve an understanding of solutions of (4.7) it thus suffices to study the system (4.8). An interesting class of solutions of (4.8) are the travelling waves, having the form $p(t, x)=P(x-\sigma t)$. The function $P: \mathbb{R} \mapsto \mathbb{R}^{2}$ must then satisfy the second order O.D.E.

$$
P^{\prime \prime}=-[A(P)+\sigma I] P^{\prime},
$$

where $A=D H$ is the Jacobian matrix in (4.6) and $I$ denotes the $2 \times 2$ identity matrix.

In the following subsections we study the existence and stability of these travelling wave solutions, and discuss their significance in connection with the vanishing viscosity limit.

\subsection{Periodic viscous travelling waves.}

Integrating the equation (4.9) once, we obtain

$$
P^{\prime}=(H(\bar{P})+\sigma \bar{P})-(H(P)+\sigma P),
$$

where $\bar{P}=\left(\bar{p}_{1}, \bar{p}_{2}\right)$ is some constant vector. We are particularly interested in periodic solutions of the O.D.E.

$$
\left\{\begin{array}{l}
p_{1}^{\prime}=\left(\bar{p}_{1} \bar{p}_{2}+\bar{p}_{1}^{2} / 2\right)-\left(p_{1} p_{2}+p_{1}^{2} / 2\right)-\sigma\left(p_{1}-\bar{p}_{1}\right) \\
p_{2}^{\prime}=\left(\bar{p}_{1} \bar{p}_{2}+\bar{p}_{2}^{2} / 2\right)-\left(p_{1} p_{2}+p_{2}^{2} / 2\right)-\sigma\left(p_{2}-\bar{p}_{2}\right)
\end{array}\right.
$$

taking values inside the elliptic region where $p_{1} p_{2}<0$. Linearizing (4.10) at the equilibrium point $\left(\bar{p}_{1}, \bar{p}_{2}\right)$ one gets

$$
\left\{\begin{array}{l}
z_{1}^{\prime}=-\left(\bar{p}_{1}+\bar{p}_{2}+\sigma\right) z_{1}-\bar{p}_{1} z_{2} \\
z_{2}^{\prime}=-\bar{p}_{2} z_{1}-\left(\bar{p}_{1}+\bar{p}_{2}+\sigma\right) z_{2}
\end{array}\right.
$$

Notice that, if one chooses $\sigma=\bar{\sigma} \doteq-\bar{p}_{1}-\bar{p}_{2}$, then the two eigenvalues

$$
\lambda_{1}, \lambda_{2}=-\left(\bar{p}_{1}+\bar{p}_{2}+\sigma\right) \pm i \sqrt{-\bar{p}_{1} \bar{p}_{2}}
$$

are purely imaginary. By the Hopf bifurcation theorem $[\mathrm{P}]$, for every $\delta>0$ sufficiently small there exists a value $\sigma=\sigma(\delta) \approx \bar{\sigma}$ such that the corresponding system (3.10) has a periodic orbit passing through the point $\left(\bar{p}_{1}+\delta, \bar{p}_{2}\right)$. More details of this construction can be found in the Appendix.

In this way, we obtain a family of periodic orbits for the system (4.10), depending on the parameters $\bar{p}_{1}, \bar{p}_{2}$ and $\delta$. If $s \mapsto\left(p_{1}(s), p_{2}(s)\right)$ is any such orbit, then

$$
\left(p_{1}(t, x), p_{2}(t, x)\right) \doteq\left(p_{1}(x-\sigma t), p_{2}(x-\sigma t)\right)
$$


yields a solution of the parabolic system (4.8) in the form of a periodic travelling wave. In turn, the functions

$$
\left(p_{1}^{\varepsilon}(t, x), p_{2}^{\varepsilon}(t, x)\right)=\left(p_{1}\left(\frac{x-\sigma t}{\varepsilon}\right), p_{2}\left(\frac{x-\sigma t}{\varepsilon}\right)\right)
$$

provide a solution to the system (4.7) with small viscosity.

We now recall that, by (4.3), the corresponding dynamic of the system is

$$
\dot{x}(t)=u_{1}^{*}+u_{2}^{*}=p_{1}\left(\frac{x-\sigma t}{\varepsilon}\right)+p_{2}\left(\frac{x-\sigma t}{\varepsilon}\right)
$$

In our construction,

$$
p_{1}+p_{2} \approx \bar{p}_{1}+\bar{p}_{2} \neq \sigma \approx-\bar{p}_{1}-\bar{p}_{2}
$$

As the viscosity parameter $\varepsilon \rightarrow 0+$, along each trajectory the controls $\left(u_{1}^{*}, u_{2}^{*}\right)=\left(p_{1}^{\varepsilon}, p_{2}^{\varepsilon}\right)$ are periodic functions of time with fixed amplitude and with period approaching zero. Because of this oscillatory behavior, there is no strong limit in $\mathbf{L}^{1}$. Yet, a weak limit exists and can be represented in terms of Young measures [S1]. These oscillatory limits can now be interpreted as chattering feedback controls. The limit trajectories cover the whole $t-x$ plane. They all have the same constant speed, determined by the weak limit of $p_{1}^{\varepsilon}+p_{2}^{\varepsilon}$.

\subsection{Instability of steady states and periodic travelling waves.}

In the elliptic region where $p_{1} p_{2}<0$, all constant solutions of (4.4) are unstable. Yet, relying on the above analysis, one may hope to recover some well-posedness for the nonlinear system (4.4) by working with measure-valued solutions: in particular, the Young measures obtained as vanishing viscosity limits of periodic travelling waves. We remark that a similar approach was considered in $[\mathrm{FL}]$. See also $[\mathrm{KY}]$ for numerical experiments in this direction. The following analysis, however, shows that viscous travelling waves of (4.8) have almost the same instability properties as the constant states. The eventual conclusion is that the use of measure-valued solutions is not of much help in connection with our ill-posed problem.

We begin our analysis by linearizing the viscous system (4.8) around a constant state $\tilde{p}=\left(\tilde{p}_{1}, \tilde{p}_{2}\right)$, assuming $\tilde{p}_{1}, \tilde{p}_{2}<0$. This yields

$$
z_{t}-A(\tilde{p}) z_{x}=z_{x x} .
$$

Working within a space of periodic functions with period $L$, this linear system can be solved by means of Fourier series. Let

$$
z(t, x)=\sum_{k} z_{k}(t) \cdot e^{i k \cdot(2 \pi x / L)}
$$


be the expansion of a periodic solution of (4.14). Then each coefficient $t \mapsto z_{k}(t) \in \mathbf{C}^{2}$ is a complex, vector valued function, satisfying the O.D.E.

$$
\frac{d z_{k}}{d t}=\left(i k \frac{2 \pi}{L} A(\tilde{p})-k^{2} \frac{4 \pi^{2}}{L^{2}} I\right) \cdot z_{k} \doteq B_{k} z_{k} .
$$

Here $I$ is the $2 \times 2$ identity matrix. Therefore

$$
z_{k}(t)=\exp \left(B_{k} t\right) z_{k}(0) .
$$

The stability of $z_{k}$ clearly depends on the spectrum of the complex valued $2 \times 2$ matrix $B_{k}$. Indeed, if $B_{k}$ has an eigenvalue $\lambda\left(B_{k}\right)$ with positive real part, then the norm of $z_{k}(t)$ can grow exponentially in time. By (4.6), the matrix $A=A(\tilde{p})$ has eigenvalues

$$
\lambda(A)=\tilde{p}_{1}+\tilde{p}_{2} \pm i \sqrt{\left|\tilde{p}_{1} \tilde{p}_{2}\right|} .
$$

Therefore, setting $\widetilde{L} \doteq 2 \pi / \sqrt{\left|\tilde{p}_{1} \tilde{p}_{2}\right|}$, we find

$$
\operatorname{Re}\left(\lambda\left(B_{k}\right)\right)=\operatorname{Re}\left(i k \frac{2 \pi}{L} \cdot\left(\tilde{p}_{1}+\tilde{p}_{2} \pm i \sqrt{\left|\tilde{p}_{1} \tilde{p}_{2}\right|}\right)-k^{2} \frac{4 \pi^{2}}{L^{2}}=\frac{2 k \pi}{L}\left( \pm \frac{2 \pi}{\widetilde{L}}-\frac{2 k \pi}{L}\right) .\right.
$$

Therefore, all components $z_{k}$ with $0<|k|<L / \widetilde{L}$ are unstable. In particular, if $\widetilde{L}<L$, then at least one unstable mode exists (namely: $z_{1}$ ) and the steady state solution is unstable w.r.t. spatially periodic perturbations of period $L$.

Next, we linearize the equation around a periodic travelling wave. Let $p$ be a travelling wave of the form (4.12), corresponding to a small periodic orbit bifurcating from the steady state $\bar{p}=\left(\bar{p}_{1}, \bar{p}_{2}\right)$, with an average value $\tilde{p}=\left(\tilde{p}_{1}, \tilde{p}_{2}\right)$. An infinitesimal perturbation $z$ will now satisfy the following linearized evolution equation:

$$
z_{t}-A(p) z_{x}-(z \bullet A(p)) p_{x}=z_{x x}
$$

where the symbol "•" denotes a directional derivative:

$$
z \bullet A(p) \doteq \lim _{h \rightarrow 0} \frac{A(p+h z)-A(p)}{h}
$$

We assume that $s \mapsto p(s)$ is a periodic orbit of (4.10), say passing through the point $\left(\bar{p}_{1}+\epsilon \sqrt{\left|\bar{p}_{2}\right|} \bar{p}_{2}\right.$ ), with $\sigma=\sigma(\epsilon) \approx-\left(\bar{p}_{1}+\bar{p}_{2}\right)$ (see the detailed computations in the Appendix). In the following we shall write $p=p^{(\epsilon)}$ to emphasize the dependence on the bifurcation parameter $\epsilon$ (not to be confused with the viscosity coefficient $\varepsilon$ in (4.7) and (4.13)). Call $L(\epsilon)$ the period of this orbit, as in (A.8). The time period of the travelling wave $p^{(\epsilon)}=p^{(\epsilon)}(t, x)$ in (4.12) is therefore

$$
T(\epsilon)=L(\epsilon) /|\sigma(\epsilon)| .
$$

It will thus be convenient to work within the space of periodic functions having period $N L(\epsilon)$, for some integer $N$. For a fixed $\epsilon>0$, define the linear operator $Z^{(\epsilon)}$ by setting

$$
Z^{(\epsilon)} \phi \doteq z(T(\epsilon))
$$


where $z$ is the solution of the linear Cauchy problem

$$
z_{t}-A\left(p^{(\epsilon)}\right) z_{x}-\left(z \bullet A\left(p^{(\epsilon)}\right)\right) p_{x}^{(\epsilon)}=z_{x x}, \quad z(0, x)=\phi(x) .
$$

Here $\phi$ is any periodic function with period $N L(\epsilon)$. From the standard theory of parabolic equations, it is clear that $Z^{(\epsilon)}$ is a compact linear operator on the space $\mathbf{L}^{2}\left([0, N L(\epsilon)] ; \mathbb{R}^{2}\right)$.

Proposition 1 For every $\epsilon>0$ sufficiently small, the operator $Z^{(\epsilon)}$ has an eigenvalue with strictly positive real part, on the space of periodic functions with period $N L(\epsilon)$, $N \geq 2$.

Proof. Call $Z^{(0)}$ the solution operator $z(0) \mapsto z(\widetilde{T})$ for the linear system with constant coefficients (4.14), with $\tilde{p}=\left(\bar{p}_{1}, \bar{p}_{2}\right), \widetilde{T}=2 \pi / \sqrt{\left|\bar{p}_{1}, \bar{p}_{2}\right|}$. By the analysis at (4.15), when $L=N \widetilde{L}, N \geq 2$, the operator $Z^{(0)}$ has some isolated eigenvalues with strictly positive real part. To conclude the proof, we would like to say that for $\epsilon>0$ small the operator $Z^{(\epsilon)}$ is a small perturbation of $Z^{(0)}$, hence it still has an eigenvalue with positive real part. To make this argument rigorous, we have to cope with the difficulty that the operators $Z^{(\epsilon)}$ are defined on spaces of periodic functions of period $L(\epsilon)$, changing with $\epsilon$. As a preliminary, for each $\epsilon>0$ we thus re-scale the space variable according to $\tilde{x}=(\widetilde{L} / L(\epsilon)) x$. The corresponding operators $\widetilde{Z}^{(\epsilon)}$ are then defined all on the same space of functions with period $N \widetilde{L}$. As $\epsilon \rightarrow 0$, one now has the convergence $Z^{(\epsilon)} \rightarrow Z^{(0)}$ in the operator norm. We now recall the analysis at (4.15), with $L=N \widetilde{L}$. When $N \geq 2$, the operator $Z^{(0)}$ has a point spectrum containing isolated eigenvalues with finite multiplicity and strictly positive real part. By a standard perturbation argument, the same holds for every suitably small perturbation $\widetilde{Z}^{(\epsilon)}$. This achieves the proof.

\section{Transition from Nash equilibrium to a Pareto optimum}

According to the previous analysis, the non-cooperative Nash equilibrium strategies are in general extremely unstable. In the present section we explore the possibility of some partial cooperation among the two players. For a game modelling an iterated prisoner's dilemma, in [Sm] Smale introduced a class of "good" strategies, which induce the other player to cooperate. Asymptotically for large times, the outcome of the game thus drifts away from the Nash equilibrium, approaching a Pareto optimum. It is remarkable that these strategies do not require any direct communication among the players. Aim of the present section is to show that a similar approach works well also in the context of differential games.

To keep the discussion as simple as possible, we start by looking at the game with payoffs

$$
\begin{aligned}
& Y_{1}\left(u_{1}, u_{2}\right)=p_{1}\left(u_{1}+u_{2}\right)-u_{1}^{2} / 2, \\
& Y_{2}\left(u_{1}, u_{2}\right)=p_{2}\left(u_{1}+u_{2}\right)-u_{2}^{2} / 2,
\end{aligned}
$$




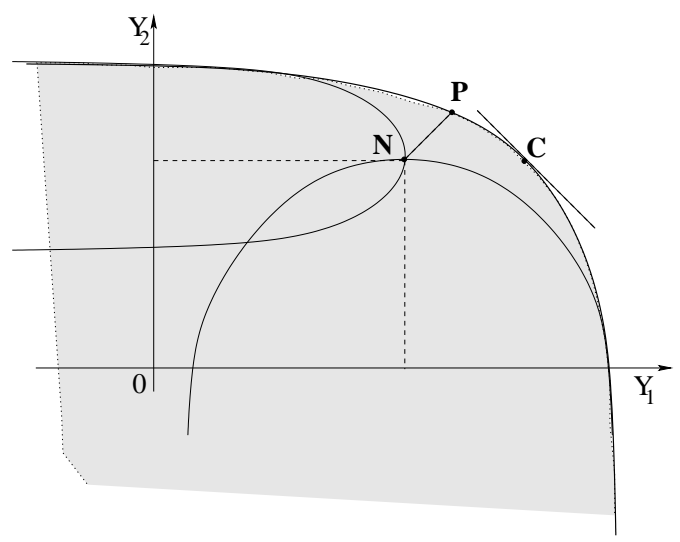

Figure 1: Semi-cooperative strategy: $\mathbf{N}$ is the Nash equilibrium, $\mathbf{C}$ is full cooperation and $\mathbf{P}$ is the semi-cooperative Pareto optimum with fairness condition.

where $p_{1}, p_{2} \in \mathbb{R}$ are given constants. Notice that this is not a differential game. The players simply choose numbers $u_{1}, u_{2}$ and achieve the payoffs $Y_{1}, Y_{2}$, respectively. Following [A], we say that the pair $\left(u_{1}^{*}, u_{2}^{*}\right) \in \mathbb{R}^{2}$ achieves a Pareto optimum if there exists no other pair $\left(u_{1}, u_{2}\right)$ such that

$$
\begin{aligned}
& Y_{1}\left(u_{1}, u_{2}\right)>Y_{1}\left(u_{1}^{*}, u_{2}^{*}\right), \\
& Y_{2}\left(u_{1}, u_{2}\right)>Y_{2}\left(u_{1}^{*}, u_{2}^{*}\right) .
\end{aligned}
$$

In other words, the two players cannot simultaneously improve their gains by implementing any other strategy. It is well known that Pareto optima are not unique. Indeed, for every $s>0$ the strategies

$$
U_{1}^{P}=p_{1}+\frac{p_{2}}{s}, \quad U_{2}^{P}=s p_{1}+p_{2}
$$

yield a Pareto optimum, maximizing the combined payoff $s Y_{1}+Y_{2}$. To remove this ambiguity, one may invoke some fairness condition and single out the unique Pareto optimum which brings an equal improvement to both players compared with the Nash strategy. Namely, let us denote by

$$
U_{i}^{N}=p_{i}, \quad Y_{i}^{N}=p_{i} \cdot\left(p_{1}+p_{2}\right)-\frac{p_{i}^{2}}{2}
$$

respectively the constant controls and the payoffs corresponding to the Nash equilibrium. A unique Pareto optimum can then be determined by the condition

$$
Y_{1}^{P}-Y_{1}^{N}=Y_{2}^{P}-Y_{2}^{N}
$$

In the following, we assume that some Pareto optimum has been chosen, and write

$$
U_{i}^{P}, \quad Y_{i}^{P}=p_{i} \cdot\left(U_{1}^{P}+U_{2}^{P}\right)-\frac{\left(U_{i}^{P}\right)^{2}}{2},
$$


respectively for the controls and the payoffs of the two players. Instead of (5.2), we shall only require the much weaker condition

$$
Y_{1}^{P}>Y_{1}^{N}, \quad Y_{2}^{P}>Y_{2}^{N} .
$$

Otherwise stated, both players gain something in the transition from the Nash equilibrium to the Pareto optimum.

We now consider the corresponding game in continuous time, with payoffs

$$
J_{i}=\frac{1}{T} \int_{0}^{T}\left\{p_{i}\left(u_{1}+u_{2}\right)-\frac{u_{i}^{2}(t)}{2}\right\} d t .
$$

Taking the point of view of the first player, our main interest is to devise strategies of the form

$$
\dot{u}_{1}=\phi_{1}\left(u_{1}, u_{2}\right),
$$

which will eventually steer the game toward the Pareto optimum. According to (5.5), the response $u_{1}$ thus changes in time. It is determined by an O.D.E. whose right hand side depends continuously on $u_{1}$ and $u_{2}$.

In analogy with $[\mathrm{Sm}]$, we say that the Lipschitz continuous function $\phi_{1}$ in (5.5) is a good strategy for player 1 if the following three conditions hold.

(C1) If the gain of the first player is smaller than what he gets by playing the Nash strategy, then he leans back toward $U_{1}^{N}$. Namely

$$
\phi_{1}\left(u_{1}, u_{2}\right) \cdot\left(u_{1}-U_{1}^{N}\right)<0 \quad \text { if } \quad Y_{1}\left(u_{1}, u_{2}\right)<Y_{1}^{N} .
$$

(C2) If the second player is gaining more than his due profit $Y_{2}^{P}$, then the first player should again lean back toward $U_{2}^{N}$. More precisely

$$
\phi_{1}\left(u_{1}, u_{2}\right) \cdot\left(u_{1}-U_{1}^{N}\right)<0 \quad \text { if } \quad Y_{2}\left(u_{1}, u_{2}\right)>Y_{2}^{P} .
$$

(C3) If the second player is cooperating, then the first player should approach the Pareto strategy. More precisely:

$$
\phi_{1}\left(u_{1}, u_{2}\right) \cdot\left(u_{1}-U_{1}^{P}\right)<0 \quad \text { if } \quad\left(Y_{1}\left(u_{1}, u_{2}\right), Y_{2}\left(u_{1}, u_{2}\right)\right) \in \Omega_{1},
$$

where

$\Omega_{1} \doteq\left\{\left(Y_{1}, Y_{2}\right) ; \quad Y_{1}>Y_{1}^{N}, \quad \frac{Y_{1}-Y_{1}^{N}}{Y_{1}^{P}-Y_{1}^{N}} \geq \frac{Y_{2}-Y_{2}^{N}}{Y_{2}^{P}-Y_{2}^{N}}\right\} \cup\left\{\theta Y^{P}+(1-\theta) Y^{N} ; 0<\theta<1\right\}$.

Notice that the first two conditions say that player 1 should play "tough" whenever the other player is taking advantage of him. The last condition implies that he should play "soft" when the game goes in his favor or when the other player is cooperating. 
For a given Pareto optimum $\left(U_{1}^{P}, U_{2}^{P}\right)$, the definition of a good strategy for player 2 is completely analogous. One can now envision a situation where each player adopts a partially cooperative strategy, based on the behavior of the other player. The game thus evolves according to

$$
\left\{\begin{array}{l}
\dot{u}_{1}=\phi_{1}\left(u_{1}, u_{2}\right) \\
\dot{u}_{2}=\phi_{2}\left(u_{1}, u_{2}\right)
\end{array}\right.
$$

The next result shows that if both players adopt good strategies, then the outcome of the game will approach the Pareto optimum.

Theorem 1 Let a Pareto optimal pair $\left(U_{1}^{P}, U_{2}^{P}\right)$ be given, satisfying (5.3). Assume that the functions $\phi_{1}, \phi_{2}$ are "good strategies", so that the corresponding conditions (C1), (C2) and (C3) hold. Then the point $\left(U_{1}^{P}, U_{2}^{P}\right)$ is an equilibrium for the dynamical system (5.6). Its basin of attraction contains the entire open rectangle

$$
\Gamma \doteq\left\{\left(u_{1}, u_{2}\right) ; \quad u_{1} \in\right] U_{1}^{N}, U_{1}^{P}\left[, \quad u_{2} \in\right] U_{2}^{N}, U_{2}^{P}[\}
$$

Proof. To fix the ideas, we consider the case where $p_{1}, p_{2}>0$. If $p_{1}<0$ or $p_{2}<0$, the proof is entirely similar. Notice that, in the degenerate case where $p_{2}=0$, the Nash equilibrium coincides with the unique Pareto optimum: $\left(U_{1}^{N}, U_{N}^{2}\right)=\left(U_{1}^{P}, U_{P}^{2}\right)=\left(p_{1}, 0\right)$, and the analysis is trivial.

From the assumption $p_{1}, p_{2}>0$ we deduce that, for some $s>0$,

$$
p_{1}+\frac{p_{2}}{s}=U_{1}^{P}>U_{1}^{N}=p_{1}, \quad s p_{1}+p_{2}=U_{2}^{P}>U_{2}^{N}=p_{2} .
$$

The flow of (5.6) on the rectangle $\Gamma$ and on its image under the map $\left(u_{1}, u_{2}\right) \mapsto\left(Y_{1}, Y_{2}\right)$ is illustrated in Figure 2. For convenience we write

$$
N \doteq\left(U_{1}^{N}, U_{2}^{N}\right), \quad P \doteq\left(U_{1}^{P}, U_{2}^{P}\right), \quad A \doteq\left(U_{1}^{N}, U_{2}^{P}\right) \quad B \doteq\left(U_{1}^{P}, U_{2}^{N}\right) .
$$

The positive invariance of $\Gamma$ is easily checked:

- On the segment $\overline{N A}$ joining $N$ with $A$, the payoff of player 1 is $Y_{1}>Y_{1}^{N}$, while $Y_{2}<Y_{2}^{N}$. Hence by (C3) it follows $\dot{u}_{1}>0$, while (C1) implies $\dot{u}_{2}<0$. Across this part of the boundary, the flow thus moves inward, as indicated in Figure 2.

- On the segment $\overline{N B}$ one has $Y_{2}>Y_{2}^{N}$, while $Y_{1}<Y_{1}^{N}$. Therefore $\dot{u}_{2}>0$ and $\dot{u}_{1}<0$. Again, the flow points inward.

- On the segment $\overline{P A}$ one has $Y_{1}>Y_{1}^{N}$ while $Y_{2}<Y_{2}^{N}$. Hence

$$
\frac{Y_{1}-Y_{1}^{N}}{Y_{1}^{P}-Y_{1}^{N}}>0>\frac{Y_{2}-Y_{2}^{N}}{Y_{2}^{P}-Y_{2}^{N}} .
$$

By condition (C3) it follows $\dot{u}_{1}>0$ while (C1) implies $\dot{u}_{2}<0$. The flow thus points inward as indicated in Figure 2. 

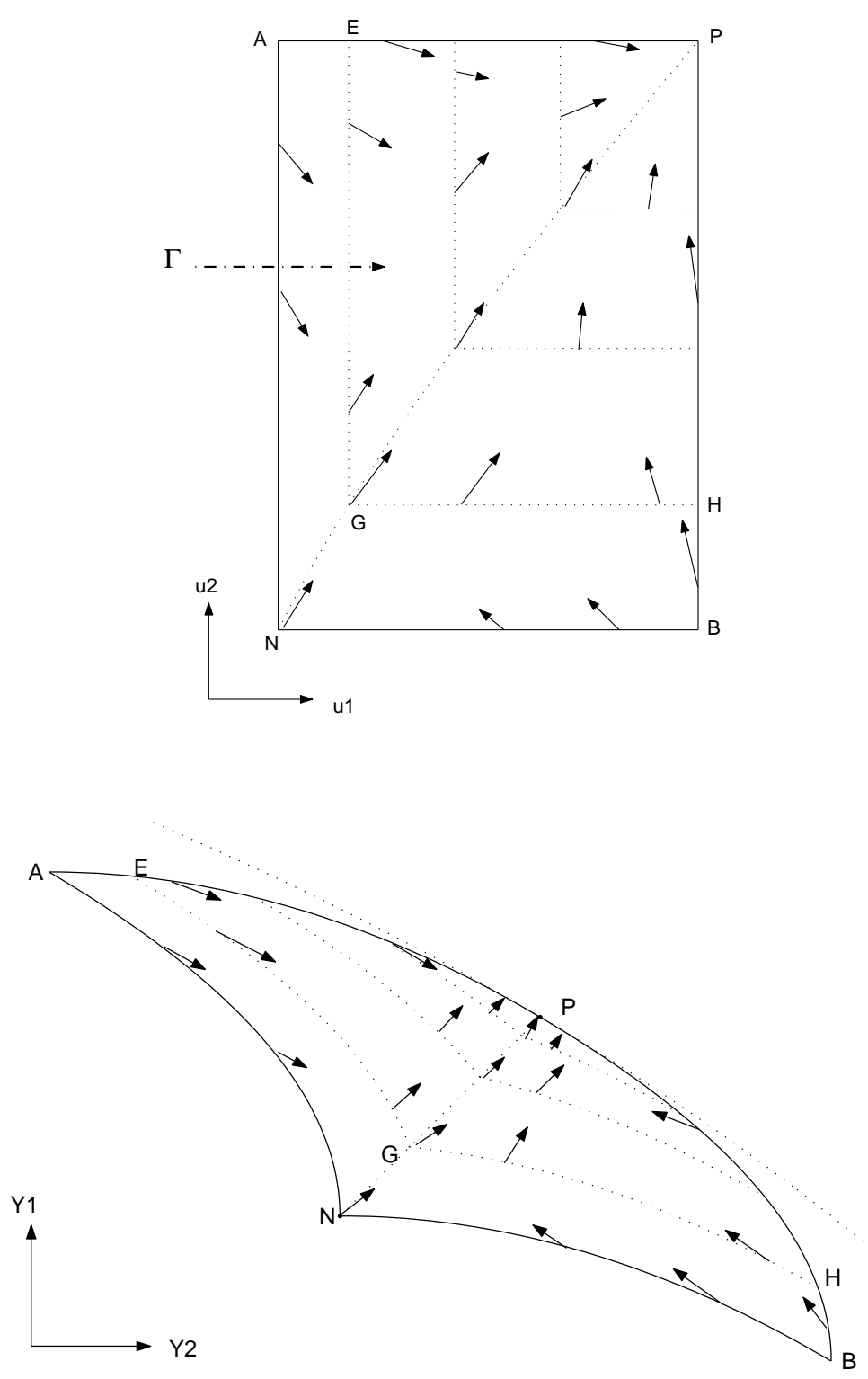

Figure 2: The flow the O.D.E. in $u$-coordinates (above) and in $Y$-coordinate (below). Here, the same letters are used to denote a point in the $u$-domain and its image in the $Y$-domain. 
- On $\overline{P B}$ we have $Y_{2}>Y_{2}^{N}$ while $Y_{1}<Y_{1}^{N}$. Hence $\dot{u}_{2}>0, \dot{u}_{1}<0$ and the flow points inward.

Looking at the signs of the components $\phi_{1}, \phi_{2}$ along the boundary of the rectangle $\Gamma$, by continuity we conclude that $N$ and $P$ are equilibrium points for the system (5.6). On the other hand, at $A$ and at $B$ the flow is strictly inward. We remark that, since there are no equilibrium points inside $\Gamma$, the flow cannot contain any periodic orbits either.

It remains to show that every trajectory starting inside $\Gamma$ eventually approaches the Pareto optimum $P=\left(U_{1}^{P}, U_{2}^{P}\right)$. Toward this goal, consider the curve $\gamma: \theta \mapsto\left(u_{1}(\theta), u_{2}(\theta)\right)$ implicitly defined as

$$
Y\left(u_{1}(\theta), u_{2}(\theta)\right)=\theta Y^{P}+(1-\theta) Y^{N} .
$$

By the implicit function theorem, this is certainly well defined, at least when $\theta \in[0, \varepsilon]$ with $\varepsilon>0$ small. We now observe that, on the segment

$$
u_{1}=u_{1}(\theta), \quad u_{2} \in\left[u_{2}(\theta), U_{2}^{P}\right]
$$

by (C3) there holds $\dot{u}_{1}>0$. On the other hand, on the segment

$$
u_{2}=u_{2}(\theta), \quad u_{1} \in\left[u_{1}(\theta), U_{1}^{P}\right]
$$

again by (C3) one has $\dot{u}_{2}>0$. The above shows that, for every $\theta>0$ sufficiently small, the rectangular region

$$
\Gamma_{\theta} \doteq\left\{\left(u_{1}, u_{2}\right) ; \quad u_{1} \in\right] u_{1}(\theta), U_{1}^{P}\left[, \quad u_{2} \in\right] u_{2}(\theta), U_{2}^{P}[\}
$$

is positively invariant for the flow (5.6). Therefore, no trajectory starting inside $\Gamma$ can approach the Nash equilibrium $U^{N}$ as $t \rightarrow \infty$.

Now consider any trajectory $t \mapsto u(t)=\left(u_{1}(t), u_{2}(t)\right)$ taking values inside $\Gamma$. Consider its $\omega$-limit set, i.e. the set of all points $\bar{u}$ such that there exists a sequence of times $t_{n} \rightarrow \infty$ such that

$$
\lim _{n \rightarrow \infty} u\left(t_{n}\right)=\bar{u}
$$

By the previous analysis, this set cannot contain the Nash equilibrium $U^{N}$. Moreover, it cannot contain any periodic orbit, because in the region bounded by a periodic orbit the vector field $\phi$ should have a zero, and no such zeroes exist inside $\Gamma$. Since we are dealing with a dynamical system on the plane, we can now use the Poincare-Bendixon theorem (see $[\mathrm{HS}],[\mathrm{P}]$ ) and conclude that this $\omega$-limit set consists of the single point $U^{P}$, completing the proof.

If both players adopt a strategy tending to the same Pareto optimum, by the previous theorem the game will asymptotically converge to that point, as expected. In this connection, two natural questions arise: 
1. What happens if the two players adopt "good" strategies, but geared toward different Pareto optima? For example, the first player might consider fair the payoffs $\left(Y_{1}^{P}, Y_{2}^{P}\right)$, while the second player might consider fair another Pareto optimum, say $\left(\widetilde{Y}_{1}^{P}, \tilde{Y}_{2}^{P}\right)$.

2. Assume that the first player adopts a good strategy, expecting the payoffs $\left(Y_{1}^{P}, Y_{2}^{P}\right)$. Can the second player use a smarter strategy and gain more than $Y_{2}^{P}$, on average?

We start by discussing the second problem. To fix the ideas, assume $p_{1}, p_{2}>0$. Let $\dot{u}_{1}=\phi_{1}\left(u_{1}, u_{2}\right)$ be a good strategy for player 1 , satisfying the conditions $(\mathrm{C} 1)-(\mathrm{C} 3)$. If player 2 cooperates, after a while the system will thus move toward the Pareto optimum $P$.

At this stage, however, player 2 can quickly change his control, setting it close to the Nash equilibrium. The payoffs $\left(Y_{1}, Y_{2}\right)$ will thus move from a point $Y^{D}$ close to the Pareto optimum to a point $Y^{E}$, with $Y_{2}^{E}>Y_{2}^{P}, Y_{1}^{E}<Y_{1}^{P}$. Of course, player 1 does not like this behavior, so he will decrease $u_{1}$, approaching the Nash equilibrium $U_{1}^{N}$. As a consequence, the payoff of the second player will decrease, continuously in time. When the payoffs reach a point $Y^{F}$ such that $Y_{2}^{F}=Y_{2}^{P}$, player 2 decides to cooperate again, setting his control back up to $U_{2}^{P}$, reaching the point $\mathrm{G}$. The new payoffs $\left(Y_{1}^{G}, Y_{2}^{G}\right)$ are now in favor of the first player, which is again willing to cooperate. The point $\left(Y_{1}^{D}, Y_{2}^{D}\right)$ close to a Pareto optimum is reached once again, and the whole cycle can be repeated. See Figure 3 for an illustration.

Notice that in this periodic strategy, the transitions from $Y^{D}$ to $Y^{E}$, and from $Y^{F}$ to $Y^{G}$ are very quick. Along the arc connecting $Y^{E}$ with $Y^{F}$ player 2 gains more than his fair share $Y^{P}$, while along the arc connecting $Y^{G}$ with $Y^{D}$ player 2 gains less than $Y^{P}$. Overall, his strategy will be profitable if the time spent along the first arc is large compared with the time spend on the latter.

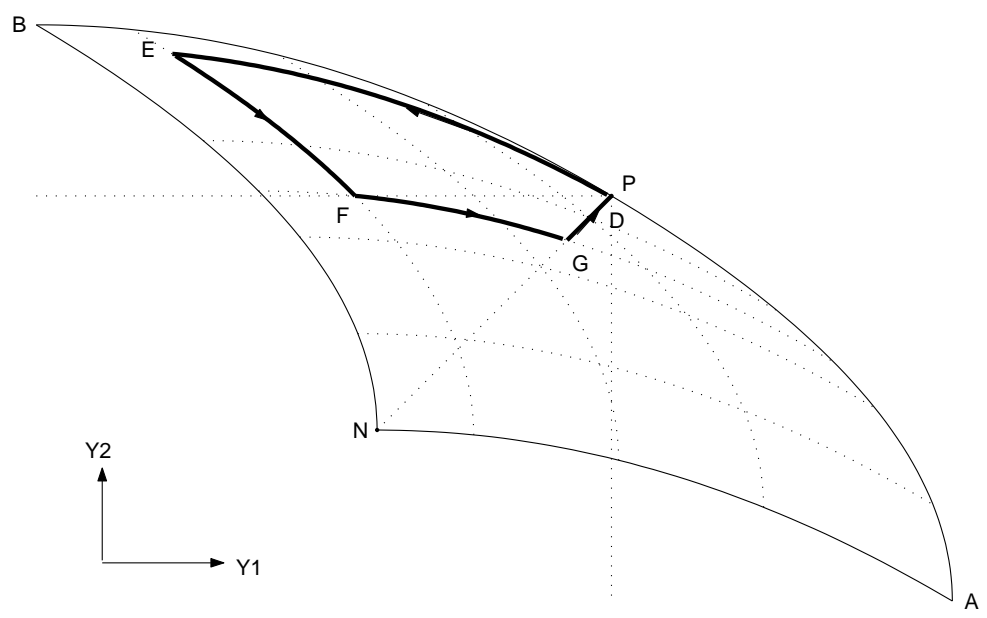

Figure 3: A possible "cheating cycle" by player 2 . 
Based on this analysis, it is now easy to suggest suitable countermeasures for the first player. In order to discourage the above behavior, the first player should quickly go back to his Nash strategy when the other player is not cooperating, and only slowly approach the Pareto optimum in the cooperative case. In other words, if the other player tries to cheat, player 1 should not be too quick in restoring cooperation.

Motivated by the previous considerations, we shall say that $\phi_{1}=\phi_{1}\left(u_{1}, u_{2}\right)$ is a smart strategy for player 1 if the conditions $(\mathrm{C} 1),(\mathrm{C} 2),(\mathrm{C} 3)$ are satisfied together with the additional condition

(C4)

$$
\phi_{1}\left(u_{1}, u_{2}\right) \leq c\left[Y_{2}^{P}-Y_{2}\left(u_{1}, u_{2}\right)\right], \text { for some constant } c>0 \text { and all } u_{1}, u_{2} \text {. }
$$

The following theorem shows that, if player 1 adopts a smart strategy, then in the long run player 2 cannot achieve anything more than $Y_{2}^{P}$.

Theorem 2 Let a Pareto optimum $\left(Y_{1}^{P}, Y_{2}^{P}\right)$ be given, satisfying (5.3). Assume that player 1 adopts a corresponding smart strategy satisfying conditions (C1), (C2), (C3) and (C4). Then, for any strategy $u_{2}=u_{2}(t)$ adopted by player 2, one has

$$
\limsup _{T \rightarrow \infty} \frac{1}{T} \int_{0}^{T} Y_{2}\left(u_{1}(t), u_{2}(t)\right) d t \leq Y_{2}^{P}
$$

Proof. By the condition (C4) we have

$$
Y_{1}(t)-Y_{1}^{P} \leq-\frac{1}{c} \dot{u}_{2}
$$

In turn this yields

$$
\int_{0}^{T}\left(Y_{1}\left(u_{1}, u_{2}\right)-Y_{1}^{P}\right) d s \leq-\frac{1}{c} \int_{0}^{T} \dot{u}_{2}(t) d t \leq \frac{u_{2}(0)-u_{2}(T)}{c} .
$$

Since $u_{1}(t) \in\left[U_{1}^{N}, U_{1}^{P}\right]$ for all $t$, letting $T \rightarrow \infty$ we obtain

$$
\limsup _{T \rightarrow \infty} \frac{1}{T} \int_{0}^{T}\left(Y_{2}(s)-Y_{2}^{P}\right) d s \leq \limsup _{T \rightarrow \infty} \frac{u_{2}(0)-u_{2}(T)}{c T}=0
$$

completing the proof.

We now come back to the first question. Assume that both players adopt smart strategies, but geared toward different Pareto optima, say $\left(Y_{1}^{P}, Y_{2}^{P}\right)$ and $\left(\widetilde{Y}_{1}^{P}, \widetilde{Y}_{2}^{P}\right)$ respectively. In the light of the above theorem, the average payoff of each player will then be no better that what the other player regards as "fair". More precisely, assume that

$$
Y_{1}^{P}>\tilde{Y}_{1}^{P}, \quad Y_{2}^{P}<\tilde{Y}_{2}^{P} .
$$


Then

$\limsup _{T \rightarrow \infty} \frac{1}{T} \int_{0}^{T} Y_{1}\left(u_{1}(t), u_{2}(t)\right) d t \leq \tilde{Y}_{1}^{P}, \quad \quad \limsup _{T \rightarrow \infty} \frac{1}{T} \int_{0}^{T} Y_{2}\left(u_{1}(t), u_{2}(t)\right) d t \leq Y_{2}^{P}$.

In fact, failure to agree on what the "fair" Pareto optimum should be may well result in payoffs which are very close to the Nash equilibrium.

In the remainder of this section we sketch a heuristic argument, based on the previous analysis, that motivates the use of semi-cooperative strategies. Consider the differential game

$$
\dot{x}=f_{0}(x)+u_{1}+u_{2}
$$

with payoffs

$$
J_{i}=g_{i}(x(T))-\int_{0}^{T} \frac{u_{i}^{2}(t)}{2} d t .
$$

Call $V_{i}(t, x)$ the value functions, assumed to be sufficiently smooth. By the dynamic programming principle, at a given time $\tau$, the $i$-th player seeks to maximize the quantity

$$
\begin{aligned}
V_{i}(\tau+\delta, x(\tau+\delta))-\int_{\tau}^{\tau+\delta} \frac{u_{i}^{2}}{2} d t \approx & V_{i}(\tau, x(\tau))+\delta\left[V_{i, t}(\tau, x(\tau))+f_{0}(x(\tau)) \cdot V_{i, x}(\tau, x(\tau))\right] \\
& +\int_{\tau}^{\tau+\delta}\left\{V_{i, x}\left((\tau, x(\tau)) \cdot\left(u_{1}+u_{2}\right)-\frac{u_{i}^{2}}{2}\right\} d t\right.
\end{aligned}
$$

Since the first two terms on the right hand side of (5.10) do not depend on $u_{1}, u_{2}$, the short-term problem for the $i$-th player is to maximize the last integral, over the interval $[\tau, \tau+\delta]$. This integral has the same form as form as (5.4), with $V_{i, x}$ in place of $p_{i}$ and the interval $[\tau, \tau+\delta]$ replacing $[0, T]$. One can thus envision a situation where the players implement "good" strategies of the form

$$
\left\{\begin{array}{l}
\dot{u}_{1}=\varepsilon^{-1} \phi_{1}\left(u_{1}, u_{2}\right) \\
\dot{u}_{2}=\varepsilon^{-1} \phi_{2}\left(u_{1}, u_{2}\right) .
\end{array}\right.
$$

Here $\varepsilon>0$ is a small parameter related to the "response time", i.e. the time it takes to each player to adjust its control $u_{i}$ to the strategy of the other player. By Theorem 1, if we fix any $T>0$ and let $\varepsilon \rightarrow 0$, the solutions of (5.11) will approach the Pareto optimum $\left(U_{1}^{P}, U_{2}^{P}\right)$ within the time interval $[0, T]$. If $\varepsilon<<\delta$, it is thus reasonable to consider a situation where, given the values $p_{1}=V_{1, x}$ and $p_{2}=V_{2, x}$, both players immediately implement the Pareto optimal strategies $\left(U_{1}^{P}, U_{2}^{P}\right)$, say in connection with the unique Pareto optimum satisfying the "fairness" condition (5.2). This approximation is appropriate whenever the time scale $\varepsilon$, measuring the rate of convergence to a local Pareto optimum, is substantially smaller than the time scale at which the gradients $V_{i, x}$ vary along a trajectory. 


\section{Weak hyperbolicity}

In the previous section we defined a semi-cooperative pair of strategies for the two players, achieving a Pareto optimum. When these strategies are implemented, the value functions will satisfy a different system of Hamilton-Jacobi equations. We now study this system in more detail and prove that it is always weakly hyperbolic.

Consider a differential game on $\mathbb{R}^{m}$ with dynamics

$$
\dot{x}=u_{1}+u_{2}, \quad x(\tau)=y,
$$

and payoffs

$$
J_{i}=g_{i}(x(T))-\int_{\tau}^{T} h_{i}\left(u_{i}(t)\right) d t \quad i=1,2 .
$$

As usual, we assume that $h_{1}, h_{2}$ are smooth and strictly convex. Given two gradient vectors $p_{i}=\left(p_{i 1}, \ldots, p_{i m}\right) \in \mathbb{R}^{m}, i=1,2$, let us define the instantaneous gain functionals $Y_{1}, Y_{2}$ as

$$
\begin{aligned}
& Y_{1}\left(p_{1}, p_{2}, u_{1}, u_{2}\right)=p_{1} \cdot\left(u_{1}+u_{2}\right)-h_{1}\left(u_{1}\right), \\
& Y_{2}\left(p_{1}, p_{2}, u_{1}, u_{2}\right)=p_{2} \cdot\left(u_{1}+u_{2}\right)-h_{2}\left(u_{2}\right) .
\end{aligned}
$$

Here and in the sequel, the dot denotes an inner product.

A natural way to construct a Pareto optimum is to maximize the combined payoff $s Y_{1}+Y_{2}$ for some $s>0$. More precisely, given $p_{1}, p_{2} \in \mathbb{R}$ and $s>0$, a pair of Pareto optimal controls $U_{i}^{P}\left(p_{1}, p_{2}, s\right), i=1,2$ is defined by setting

$$
\begin{aligned}
& s Y_{1}\left(p_{1}, p_{2}, U_{1}^{P}\left(p_{1}, p_{2}, s\right), U_{2}^{P}\left(p_{1}, p_{2}, s\right)\right)+Y_{2}\left(p_{1}, p_{2}, U_{1}^{P}\left(p_{1}, p_{2}, s\right), U_{2}^{P}\left(p_{1}, p_{2}, s\right)\right) \\
& \quad=\max _{u_{1}, u_{2}}\left\{s Y_{1}\left(p_{1}, p_{2}, u_{1}, u_{2}\right)+Y_{2}\left(p_{1}, p_{2}, u_{1}, u_{2}\right)\right\} .
\end{aligned}
$$

It will be convenient to write

$$
Y_{i}^{P}\left(p_{1}, p_{2}, s\right) \doteq Y_{i}\left(p_{1}, p_{2}, U_{1}^{P}\left(p_{1}, p_{2}, s\right), U_{2}^{P}\left(p_{1}, p_{2}, s\right)\right)
$$

to emphasize the dependence on $s$ of these instantaneous gain functions, when both players implement Pareto optimal strategies.

Next, assume that the players adopt feedback strategies of the form

$$
u_{1}=u_{1}^{*}\left(p_{1}, p_{2}\right), \quad u_{2}=u_{1}^{*}\left(p_{1}, p_{2}\right),
$$

where $p_{1}=\nabla_{x} V_{1}, p_{2}=\nabla_{x} V_{2}$ are the gradients of their value functions. These functions $V_{1}, V_{2}$ will then satisfy the system of $\mathrm{H}-\mathrm{J}$ equations

$$
\left\{\begin{array}{l}
V_{1, t}+H_{1}\left(\nabla_{x} V_{1}, \nabla_{x} V_{2}\right)=0, \\
V_{2, t}+H_{2}\left(\nabla_{x} V_{1}, \nabla_{x} V_{2}\right)=0,
\end{array}\right.
$$


with

$$
\begin{aligned}
& H_{1}\left(p_{1}, p_{2}\right)=Y_{1}\left(p_{1}, p_{2}, u_{1}^{*}\left(p_{1}, p_{2}\right), u_{2}^{*}\left(p_{1}, p_{2}\right)\right), \\
& H_{2}\left(p_{1}, p_{2}\right)=Y_{2}\left(p_{1}, p_{2}, u_{1}^{*}\left(p_{1}, p_{2}\right), u_{2}^{*}\left(p_{1}, p_{2}\right)\right) .
\end{aligned}
$$

We now show that the system (6.6) is weakly hyperbolic for a very general class of strategies $u_{i}^{*}\left(p_{1}, p_{2}\right)$, under the only assumption that they achieve Pareto optima. In particular, this includes the semi-cooperative strategy $\left(u_{1}^{*}, u_{2}^{*}\right)=\left(U_{1}^{P}, U_{2}^{P}\right)$ considered in the previous section.

Theorem 3 As the gradients $\left(p_{1}, p_{2}\right)$ of the value functions range in an open region $\Omega \subseteq \mathbb{R}^{2 m}$, assume that the players adopt Pareto optimal strategies of the form

$$
u_{i}^{*}\left(p_{1}, p_{2}\right)=U_{i}^{P}\left(p_{1}, p_{2}, s\left(p_{1}, p_{2}\right)\right) \quad i=1,2,
$$

for some smooth function $s=s\left(p_{1}, p_{2}\right)$. Then the system (6.6) is weakly hyperbolic on the domain $\Omega$.

Proof. For clarity of exposition, we first give a proof in the one-dimensional case, then extend the arguments to several space dimensions. Let $\xi \mapsto k_{i}(\xi)$ be the increasing function defined by

$$
k_{i}(\xi)=\arg \max _{\omega}\left\{\xi \cdot \omega-h_{i}(\omega)\right\}
$$

Of course, this implies

$$
h_{i}^{\prime}\left(k_{i}(\xi)\right)=\xi
$$

Notice that the Nash equilibrium strategies are

$$
u_{1}^{N}\left(p_{1}\right) \doteq k_{1}\left(p_{1}\right), \quad u_{2}^{N}\left(p_{2}\right) \doteq k_{2}\left(p_{2}\right) .
$$

For any given $\left(p_{1}, p_{2}\right) \in \Omega$, the assumption of Pareto optimality (6.8) yields

$$
u_{1}^{*}\left(p_{1}, p_{2}\right)=k_{1}\left(p_{1}+p_{2} / s\right), \quad u_{2}^{*}\left(p_{1}, p_{2}\right)=k_{2}\left(p_{2}+s p_{1}\right),
$$

with $s=s\left(p_{1}, p_{2}\right)$. By the optimality condition (6.4), at the point $\left(p_{1}, p_{2}, U_{1}^{P}(s), U_{2}^{P}(s)\right)$ one has

$$
s \frac{\partial Y_{1}}{\partial u_{1}}+\frac{\partial Y_{2}}{\partial u_{1}}=s \frac{\partial Y_{1}}{\partial u_{2}}+\frac{\partial Y_{2}}{\partial u_{2}}=0 .
$$

Recalling (6.5) we now write

$$
\frac{\partial Y_{1}^{P}}{\partial s}=\frac{\partial Y_{1}}{\partial u_{1}} \frac{\partial U_{1}^{P}}{\partial s}+\frac{\partial Y_{1}}{\partial u_{2}} \frac{\partial U_{2}^{P}}{\partial s}, \quad \frac{\partial Y_{2}^{P}}{\partial s}=\frac{\partial Y_{2}}{\partial u_{1}} \frac{\partial U_{1}^{P}}{\partial s}+\frac{\partial Y_{2}}{\partial u_{2}} \frac{\partial U_{2}^{P}}{\partial s} .
$$

Using (6.13) we obtain the identity

$$
\frac{\partial Y_{1}^{P}}{\partial s}=-\frac{1}{s} \frac{\partial Y_{2}^{P}}{\partial s}
$$


By (6.12), the Jacobian matrix $A\left(p_{1}, p_{2}\right)$ for the system (6.7) is precisely the Jacobian of the map

$$
\left(p_{1}, p_{2}\right) \mapsto\left(Y_{1}^{P}, Y_{2}^{P}\right)=\left(\begin{array}{c}
p_{1}\left[k_{1}\left(p_{1}+p_{2} / s\right)+k_{2}\left(p_{2}+s p_{1}\right)\right]-h_{1}\left(k_{1}\left(p_{1}+p_{2} / s\right)\right) \\
p_{2}\left[k_{1}\left(p_{1}+p_{2} / s\right)+k_{2}\left(p_{2}+s p_{1}\right)\right]-h_{2}\left(k_{2}\left(p_{2}+s p_{1}\right)\right)
\end{array}\right)
$$

In the special case where $s$ is a constant independent of $p_{1}, p_{2}$, we find

$$
A\left(p_{1}, p_{2}\right)=\left(\begin{array}{cc}
k_{1}+k_{2}+p_{1}\left(k_{1}^{\prime}+s k_{2}^{\prime}\right)-h_{1}^{\prime} k_{1}^{\prime} & p_{1}\left(\frac{1}{s} k_{1}^{\prime}+k_{2}^{\prime}\right)-\frac{1}{s} h_{1}^{\prime} k_{1}^{\prime} \\
p_{2}\left(k_{1}^{\prime}+s k_{2}^{\prime}\right)-s h_{2}^{\prime} k_{2}^{\prime} & k_{1}+k_{2}+p_{2}\left(\frac{1}{s} k_{1}^{\prime}+k_{2}^{\prime}\right)-h_{2}^{\prime} k_{2}^{\prime}
\end{array}\right) .
$$

In the above computation, it is understood that $k_{1}, k_{1}^{\prime}$ are evaluated at $\xi=p_{1}+p_{2} / s$ while $k_{2}, k_{2}^{\prime}$ are evaluated at $\xi=p_{2}+s p_{1}$. Introducing the quantity

$$
a \doteq p_{1} k_{2}^{\prime}-\frac{1}{s^{2}} p_{2} k_{1}^{\prime}
$$

and using the identity (6.10) we obtain

$$
A\left(p_{1}, p_{2}\right)=\left(\begin{array}{cc}
k_{1}+k_{2}+s a & a \\
-s^{2} a & k_{1}+k_{2}-s a
\end{array}\right)=\left(k_{1}+k_{2}\right) I+A^{\sharp},
$$

where

$$
A^{\sharp}=\left(\begin{array}{cc}
s a & a \\
-s^{2} a & -s a
\end{array}\right)=\left(\begin{array}{c}
1 \\
-s
\end{array}\right)\left(\begin{array}{cc}
s a & a
\end{array}\right) .
$$

It is now clear that the matrix $A$ has real eigenvalues.

Next, consider the general case where $s$ varies with $p_{1}, p_{2}$, say $s=s\left(p_{1}, p_{2}\right)$. In this case we have

$$
A\left(p_{1}, p_{2}\right)=\left(k_{1}+k_{2}\right) I+A^{\sharp}+A^{b},
$$

where

$$
A^{b}=\left(\begin{array}{ll}
\frac{\partial Y_{1}^{P}}{\partial s} \frac{\partial s}{\partial p_{1}} & \frac{\partial Y_{1}^{P}}{\partial s} \frac{\partial s}{\partial p_{2}} \\
\frac{\partial Y_{2}^{P}}{\partial s} \frac{\partial s}{\partial p_{1}} & \frac{\partial Y_{2}^{P}}{\partial s} \frac{\partial s}{\partial p_{2}}
\end{array}\right)
$$

Because of the identity $(6.14), A^{b}$ can be written as a product:

$$
A^{b}=\left(\begin{array}{c}
1 \\
-s
\end{array}\right)(c, d), \quad c=\frac{\partial Y_{1}^{P}}{\partial s} \frac{\partial s}{\partial p_{1}}, \quad d=\frac{\partial Y_{1}^{P}}{\partial s} \frac{\partial s}{\partial p_{2}} .
$$

Hence

$$
A\left(p_{1}, p_{2}\right)=\left(k_{1}+k_{2}\right) I+\left(\begin{array}{c}
1 \\
-s
\end{array}\right)\left(\begin{array}{ll}
s a+c & a+d
\end{array}\right) .
$$

A direct computation shows that $A$ has two real eigenvalues

$$
\lambda_{1}=k_{1}+k_{2}, \quad \lambda_{2}=\left(k_{1}+k_{2}\right)+(c-s d),
$$


with corresponding eigenvectors

$$
r_{1}=\left(\begin{array}{c}
a+d \\
-s a-c
\end{array}\right), \quad r_{2}=\left(\begin{array}{c}
1 \\
-s
\end{array}\right) .
$$

This proves the theorem in the one dimensional case.

We now extend the proof to several space dimensions. At a given point $\left(p_{1}, p_{2}\right)=$ $\left(p_{11}, \ldots, p_{1 m}, p_{21}, \ldots, p_{2 m}\right)$, for any $\alpha=1, \ldots, m$ the matrix $A_{\alpha}$ in (3.5) has the form

$$
A_{\alpha}=\left(k_{1 \alpha}+k_{2 \alpha}\right) I+A_{\alpha}^{\sharp}+A_{\alpha}^{b},
$$

where $I$ denotes the $2 \times 2$ identity matrix and

$$
\begin{gathered}
A_{\alpha}^{\sharp}=\left(\begin{array}{cc}
s a_{\alpha} & a_{\alpha} \\
-s^{2} a_{\alpha} & -s a_{\alpha}
\end{array}\right)=\left(\begin{array}{c}
1 \\
-s
\end{array}\right)\left(\begin{array}{cc}
s a_{\alpha} & a_{\alpha}
\end{array}\right), \\
A_{\alpha}^{b}=\left(\begin{array}{cc}
c_{\alpha} & d_{\alpha} \\
-s c_{\alpha} & -s d_{\alpha}
\end{array}\right)=\left(\begin{array}{c}
1 \\
-s
\end{array}\right)\left(\begin{array}{ll}
c_{\alpha}, & d_{\alpha}
\end{array}\right),
\end{gathered}
$$

with

$$
a_{\alpha} \doteq\left(D k_{2} \cdot p_{1}\right)_{\alpha}-\frac{1}{s^{2}}\left(D k_{1} \cdot p_{2}\right)_{\alpha}, \quad c_{\alpha} \doteq \frac{\partial Y_{1}}{\partial s} \frac{\partial s}{\partial p_{1 \alpha}}, \quad d_{\alpha}=\frac{\partial Y_{1}}{\partial s} \frac{\partial s}{\partial p_{2 \alpha}}
$$

To prove the theorem, for every vector $\xi=\left(\xi_{1}, \cdots, \xi_{m}\right)$ one needs to show that the matrix $A(\xi) \doteq \sum_{\alpha} \xi_{\alpha} A_{\alpha}$ has real eigenvalues. Writing the inner products in the form

$$
\xi \cdot k_{i} \doteq \sum_{\alpha=1}^{m} \xi_{\alpha} k_{i \alpha}, \quad \xi \cdot a \doteq \sum_{\alpha=1}^{m} \xi_{\alpha} a_{\alpha},
$$

we compute

$$
A(\xi) \doteq \sum_{\alpha} \xi_{\alpha} A_{\alpha}=\left(\xi \cdot k_{1}+\xi \cdot k_{2}\right) I+A^{\sharp}(\xi)+A^{b}(\xi)
$$

where

$$
\begin{aligned}
& A^{\sharp}(\xi)=\left(\begin{array}{cc}
\xi \cdot s a & \xi \cdot a \\
-\xi \cdot s^{2} a & -\xi \cdot s a
\end{array}\right)=\left(\begin{array}{c}
1 \\
-s
\end{array}\right)\left(\begin{array}{ll}
\xi \cdot s a & \xi \cdot a
\end{array}\right),
\end{aligned}
$$

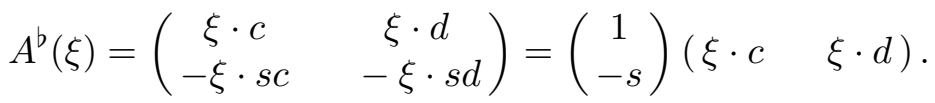

From the above expressions, it is clear that $A(\xi)$ has the two real eigenvalues

$$
\lambda_{1}(\xi)=\xi \cdot\left(k_{1}+k_{2}\right), \quad \lambda_{2}(\xi)=\xi \cdot\left(k_{1}+k_{2}+c-s d\right) .
$$

The corresponding eigenvectors are found to be

$$
r_{1}(\xi)=\left(\begin{array}{c}
\xi \cdot(a+d) \\
-\xi \cdot(s a+c)
\end{array}\right), \quad r_{2}(\xi)=\left(\begin{array}{c}
1 \\
-s
\end{array}\right) .
$$

Remark 2. The above result can be easily extended to a game of the form (3.6), with running cost functionals depending also on $x$, as in (2.3). 
Remark 3. The "semi-cooperative" Pareto optimal strategies considered at (5.2) also fit in the present framework. Indeed, they can be written in the form (6.8), choosing the parameter $s=s\left(p_{1}, p_{2}\right)$ so that

$$
\begin{aligned}
& Y_{1}\left(p_{1}, p_{2}, U_{1}^{P}(s), U_{2}^{P}(s)\right)-Y_{1}\left(p_{1}, p_{2}, U_{1}^{N}, U_{2}^{N}\right) \\
& \quad=Y_{2}\left(p_{1}, p_{2}, U_{1}^{P}(s), U_{2}^{P}(s)\right)-Y_{2}\left(p_{1}, p_{2}, U_{1}^{N}, U_{2}^{N}\right) .
\end{aligned}
$$

The Nash strategies $U_{i}^{N}$ were defined at (6.11). The map $\left(p_{1}, p_{2}\right) \mapsto s\left(p_{1}, p_{2}\right)$ is thus implicitly defined by

$$
\begin{aligned}
& p_{1}\left[k_{1}\left(p_{1}+p_{2} / s\right)+k_{2}\left(p_{2}+s p_{1}\right)-k_{1}\left(p_{1}\right)-k_{2}\left(p_{2}\right)\right]-\left[h_{1}\left(k_{1}\left(p_{1}+p_{2} / s\right)\right)-h_{1}\left(k_{1}\left(p_{1}\right)\right)\right] \\
= & p_{2}\left[k_{1}\left(p_{1}+p_{2} / s\right)+k_{2}\left(p_{2}+s p_{1}\right)-k_{1}\left(p_{1}\right)-k_{2}\left(p_{2}\right)\right]-\left[h_{2}\left(k_{2}\left(p_{2}+s p_{1}\right)\right)-h_{2}\left(k_{2}\left(p_{2}\right)\right)\right] .
\end{aligned}
$$

Remark 4. In the one-dimensional case, if $c \neq s d$, then the two eigenvalues are distinct and the system is strictly hyperbolic. Existence and stability of solutions can be obtained from the general theory of hyperbolic systems [BB], [L], [S1]. In general, however, the $\left(p_{1}, p_{2}\right)$-plane can contain one or more curves where $c=s d$. This happens when $\frac{\partial s}{\partial p_{1}}=s \frac{\partial s}{\partial p_{2}}$. Along these curves the system is only weakly hyperbolic. We do not expect to find the severe instabilities described in Section 3. Yet, in this situation the standard hyperbolic theory does not apply. It is not clear whether a local solution of the Cauchy problem still exists, if the initial datum crosses one of these curves.

\section{A Appendix: analysis of viscous periodic orbits}

In this section we compute in greater detail the viscous periodic orbits considered in Section 3. In particular, this more accurate analysis will prove the existence of solutions of the viscous system (4.8) that start arbitrarily close to a constant state and converge to a periodic viscous travelling profile as $t \rightarrow \infty$.

Two main cases will be considered, the others being similar.

CASE 1: $\bar{p}_{1}=-\bar{p}_{2}>0$. Choosing $\sigma=0$, all solutions of the O.D.E. (4.10) in the quadrant $\left\{p_{1}>0, p_{2}<0\right\}$ are periodic orbits (fig. 4). This is due to the symmetry w.r.t. the line $p_{1}+p_{2}=0$. Every such periodic orbit yields a solution of the parabolic system (4.8), constant in time and periodic w.r.t. the $x$ variable.

CASE 2: $\bar{p}_{1}>-\bar{p}_{2}>0$. Choosing $\sigma=\bar{\sigma} \doteq-\bar{p}_{1}-\bar{p}_{2}$, a subsequent analysis will show that the trajectories of the O.D.E. spiral outward from the equilibrium point 


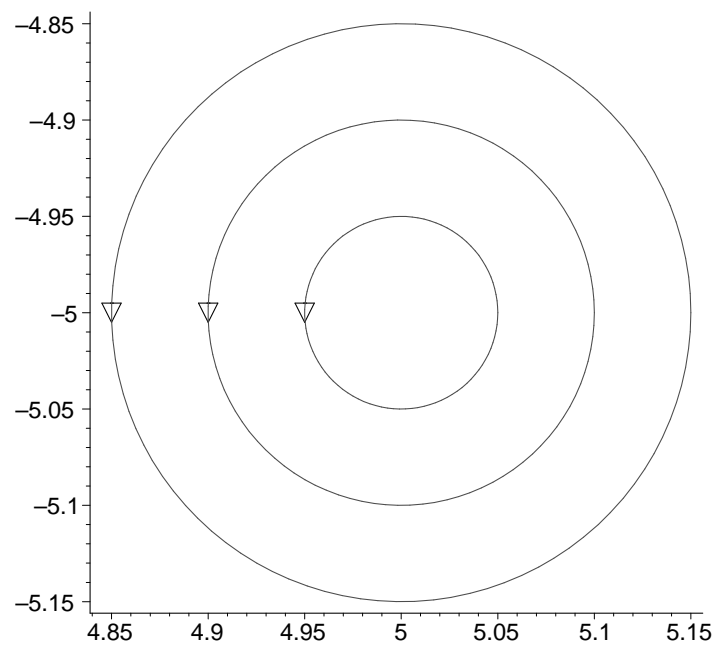

Figure 4: Periodic solutions when $p_{1}=-p_{2}$. Here the horizontal axis is $p_{1}$ and the vertical axis is $p_{2}$.

$\bar{P}=\left(\bar{p}_{1}, \bar{p}_{2}\right)$, as in fig. 5. Therefore, a Hopf bifurcation occurs as soon as $\sigma$ becomes larger than $\bar{\sigma}$ and the point $\bar{P}$ is turned into a weak sink. For all $\sigma=-\bar{p}_{1}-\bar{p}_{2}+\eta$, with $\eta \in] 0, \eta_{0}$ ] sufficiently small, the corresponding system (4.10) will thus admit a periodic orbit inside a small neighborhood of $\bar{P}$ (see fig. 6). In turn, this yields a solution of the parabolic system (4.8) in the form of a periodic travelling wave.

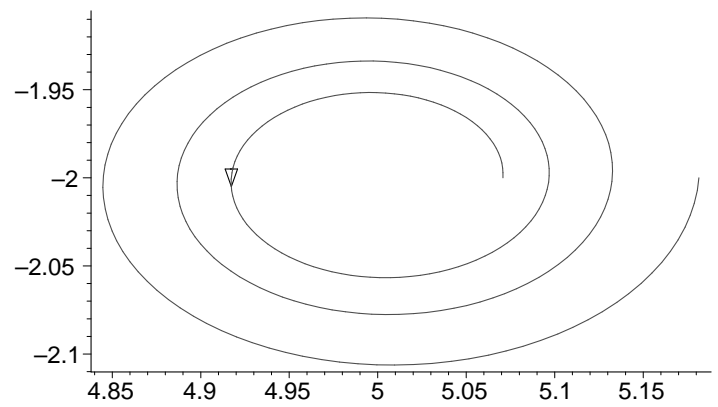

Figure 5: The critical point is a weak source. Here the horizontal axis is $p_{1}$ and the vertical axis is $p_{2}$.

The following computations substantiate the claim made in Case 2, showing that the point $\bar{P}$ is a weak source. The small quantity $\epsilon>0$ will play the role of a bifurcation parameter. The analysis needs to be a bit careful, because the lower order expansions do not suffice to reach a conclusion and we need to consider up to fourth order terms. For convenience, set $a \doteq \bar{p}_{1}$ and $b \doteq-\bar{p}_{2}$. Performing the change of variables

$$
X=\frac{\sqrt{b}}{\epsilon}\left(p_{1}-\bar{p}_{1}\right), \quad Y=\frac{\sqrt{a}}{\epsilon}\left(p_{2}-\bar{p}_{2}\right), \quad \tau=\sqrt{a b} t
$$




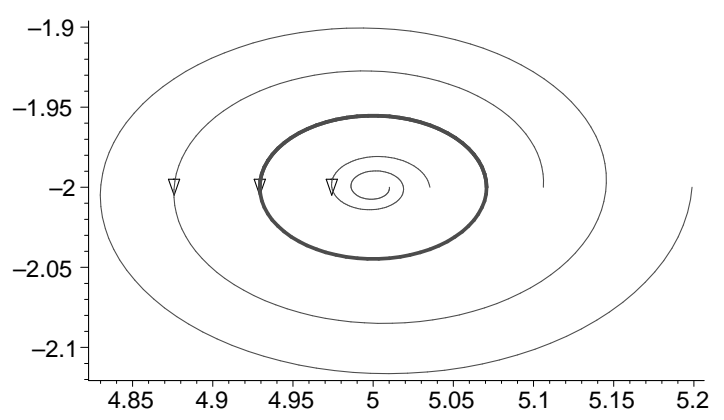

Figure 6: Hopf bifurcation: the critical point changes from weak source to weak sink, and a limit circle appears which gives a periodic solution. Here the horizontal axis is $p_{1}$ and the vertical axis is $p_{2}$.

$$
p_{1}=\bar{p}_{1}+\epsilon \frac{X}{\sqrt{b}}, \quad p_{2}=\bar{p}_{2}+\epsilon \frac{Y}{\sqrt{a}} t=\frac{\tau}{\sqrt{a b}},
$$

and denoting the differentiation w.r.t. $\tau$ by an upper dot, the system (4.10) takes the form

$$
\left\{\begin{array}{l}
\dot{X}=-Y-\frac{\epsilon}{a \sqrt{b}} X Y-\frac{\epsilon}{2 \sqrt{a} b} X^{2} \\
\dot{Y}=X-\frac{\epsilon}{\sqrt{a} b} X Y-\frac{\epsilon}{2 a \sqrt{b}} Y^{2}
\end{array}\right.
$$

It is convenient to work in polar coordinates $(r, \theta)$, with $X=r \cos \theta, Y=r \sin \theta$. Using the standard relations

$$
\dot{r}=(X \dot{X}+Y \dot{Y}) / r, \quad \dot{\theta}=(X \dot{Y}-Y \dot{X}) / r^{2},
$$

from (A.2) we get

$$
\left\{\begin{array}{l}
\dot{r}=-\epsilon r^{2} \cdot \alpha(\theta), \\
\dot{\theta}=1-\epsilon r \cdot \beta(\theta),
\end{array}\right.
$$

where $\alpha(\theta)$ and $\beta(\theta)$ can be computed directly:

$$
\begin{aligned}
\alpha(\theta) & \doteq r^{-3}\left[\frac{2 X^{2} Y+Y^{3}}{2 a \sqrt{b}}+\frac{2 X Y^{2}+X^{3}}{2 \sqrt{a} b}\right]=\frac{5 \sin \theta+\sin 3 \theta}{8 a \sqrt{b}}+\frac{5 \cos \theta-\cos 3 \theta}{8 \sqrt{a} b} \\
\beta(\theta) & \doteq r^{-3}\left[\frac{X^{2} Y}{2 \sqrt{a} b}-\frac{X Y^{2}}{2 a \sqrt{b}}\right]=\frac{\sin \theta+\sin 3 \theta}{8 \sqrt{a} b}+\frac{-\cos \theta+\cos 3 \theta}{8 a \sqrt{b}}
\end{aligned}
$$

Using (A.3) and expanding $d r / d \theta$ up to fourth order terms w.r.t. $\epsilon$, we find

$$
\frac{d r}{d \theta}=\frac{-\epsilon r^{2} A(\theta)}{1-\epsilon r^{\cdot} B(\theta)}=-\epsilon r^{2} A\left[1+(\epsilon r B)+(\epsilon r B)^{2}+(\epsilon r B)^{3}+\mathcal{O}\left(\epsilon^{4}\right)\right] .
$$

We consider the solution $r=r(\theta)$ of $(\mathrm{A} .4)$ such that $r(0)=1$. This can be written in the form

$$
r(\theta)=1+\epsilon r_{1}(\theta)+\epsilon^{2} r_{2}(\theta)+\epsilon^{3} r_{3}(\theta)+\epsilon^{4} r_{4}(\theta)+\mathcal{O}\left(\epsilon^{5}\right)
$$


where $r_{1}, r_{2}, r_{3}$ and $r_{4}$ satisfy the following O.D.E's

$$
\begin{aligned}
\frac{d}{d \theta} r_{1} & =-\alpha \\
\frac{d}{d \theta} r_{2} & =-\alpha\left[2 r_{1}+\beta\right] \\
\frac{d}{d \theta} r_{3} & =-\alpha\left[r_{1}^{2}+2 r_{2}+\beta\left[3 r_{1}+\beta\right]\right] \\
\frac{d}{d \theta} r_{4} & =-\alpha\left[2 r_{1} r_{2}+2 r_{3}+\beta\left[3 r_{2}+3 r_{1}^{2}+\beta\left[4 r_{1}+\beta\right]\right]\right]
\end{aligned}
$$

All these functions can be computed recursively, by integrations. To understand whether the trajectory is spiraling inward or outward, we need to check the sign of $r(2 \pi)$, for $\epsilon$ small. By direct computations, carried out using Maple, one finds

$$
r_{1}(2 \pi)=r_{2}(2 \pi)=r_{3}(2 \pi)=0, \quad r_{4}(2 \pi)=\frac{\pi(a-b)}{96(a b)^{7 / 2}} .
$$

Our assumption $a=\bar{p}_{1}>-\bar{p}_{2}=b$ now implies $r_{4}(2 \pi)>0$. Recalling the expansion (A.5) we conclude that, when $\sigma=-\bar{p}_{1}-\bar{p}_{2}$, the orbits of the system (4.10) are spiraling outward from the equilibrium point $\left(\bar{p}_{1}, \bar{p}_{2}\right)$, as illustrated in fig. 5 .

Next, we seek the value of the parameter $\eta=\eta(\epsilon)$ such that, setting $\sigma=-\bar{p}_{1}-\bar{p}_{2}+\eta$, the system (4.10) has a periodic solution passing through the point

$$
\left(p_{1}(0), p_{2}(0)\right)=\left(\bar{p}_{1}+\epsilon / \sqrt{b}, \bar{p}_{2}\right) .
$$

By our change of variables, this corresponds to a periodic solution of

$$
\left\{\begin{array}{l}
\dot{X}=-Y-\frac{\epsilon}{a \sqrt{b}} X Y-\frac{\epsilon}{2 \sqrt{a} b} X^{2}-\frac{\eta(\epsilon)}{\sqrt{a b}} X, \\
\dot{Y}=X-\frac{\epsilon}{\sqrt{a} b} X Y-\frac{\epsilon}{2 a \sqrt{b}} Y^{2}-\frac{\eta(\epsilon)}{\sqrt{a b}} Y,
\end{array}\right.
$$

passing through the point $(X(0), Y(0))=(1,0)$. Because of (A.6), the perturbation $\eta$ will have an expansion of the form

$$
\eta(\epsilon)=c \epsilon^{4}+\mathcal{O}\left(\epsilon^{5}\right) .
$$

The constant $c$ can be determined as follows. If (A.2) is replaced by (A.7), instead of (A.4) the evolution equation for the radius $\tilde{r}$ becomes

$$
\frac{d \tilde{r}}{d \theta}=\frac{-\epsilon \tilde{r}^{2} \cdot \alpha(\theta)-\eta(\epsilon) \tilde{r} / \sqrt{a b}}{1-\epsilon \tilde{r} \cdot \beta(\theta)}
$$

Writing again

$$
\tilde{r}=1+\epsilon \tilde{r}_{1}+\epsilon^{2} \tilde{r}_{2}+\epsilon^{3} \tilde{r}_{3}+\epsilon^{4} \tilde{r}_{4}+\mathcal{O}\left(\epsilon^{5}\right)
$$


we clearly have $r_{1}=\tilde{r}_{1}, r_{2}=\tilde{r}_{2}, r_{2}=\tilde{r}_{3}$, while

$$
\tilde{r}_{4}(2 \pi)=r_{4}(2 \pi)-2 \pi c / \sqrt{a b} .
$$

Therefore $\tilde{r}_{4}(2 \pi)=0$ provided that we choose $c=\frac{(a-b)}{192(a b)^{3}}$. By the implicit function theorem, we conclude that

$$
\eta(\epsilon)=\frac{3(a-b)}{16(a b)^{3}} \epsilon^{4}+\mathcal{O}\left(\epsilon^{5}\right)
$$

\section{A.1 Length of the period.}

We now analyze how the length of the period varies with $\epsilon$. More precisely, call $L(\epsilon)$ the period of the closed orbit of (4.10), with $\sigma=-\bar{p}_{1}-\bar{p}_{2}+\eta(\epsilon)$, passing through the point $\left(\bar{p}_{1}+\epsilon / \sqrt{b}, \bar{p}_{2}\right)$. Equivalently, $\sqrt{a b} \cdot L(\epsilon)$ is the period of the orbit of (A.7) passing through the point $(1,0)$. To compute this period, we observe that the variable $\tau=\tau(\theta)$ satisfies the differential equation

$$
\frac{d \tau}{d \theta}=\frac{1}{1-\epsilon \tilde{r} \cdot \beta(\theta)}=1+\epsilon \tilde{r} \beta+\epsilon^{2} \tilde{r}^{2} \cdot \beta^{2}+\mathcal{O}\left(\epsilon^{3}\right) .
$$

Writing $\tau(\theta)=\theta+\epsilon \tau_{1}(\theta)+\epsilon^{2} \tau_{2}(\theta)+\mathcal{O}\left(\epsilon^{3}\right)$ and observing that, by (A.6), $\tilde{r}=1+\epsilon r_{1}+$ $\epsilon^{2} r_{2}+\mathcal{O}\left(\epsilon^{3}\right)$, one obtains

$$
\frac{d \tau_{1}}{d \theta}=\beta(\theta), \quad \frac{d \tau_{2}}{d \theta}=r_{1}(\theta) \beta(\theta)+\beta(\theta)^{2} .
$$

By direct computations, again carried out with Maple, we find

$$
\tau_{1}(2 \pi)=0, \quad \tau_{2}(2 \pi)=-\frac{\pi(a+b)}{24 a^{2} b^{2}} .
$$

Hence

$$
L(\epsilon)=\frac{2 \pi}{\sqrt{a b}} \cdot\left[1-\frac{a+b}{48 a^{2} b^{2}} \epsilon^{2}+\mathcal{O}\left(\epsilon^{3}\right)\right] .
$$

According to (A.8), the period of these orbits is thus a strictly decreasing function of $\epsilon$.

\section{A.2 Average value of periodic orbits.}

We shall also need an estimate on the average value $\tilde{p}(\epsilon)=\left(\tilde{p}_{1}(\epsilon), \tilde{p}_{2}(\epsilon)\right)$ of this periodic orbit. Denoting by $L=L(\epsilon)$ the length of the period in (A.8) and recalling (A.1), we compute

$$
\tilde{p}_{1}(\epsilon)=\bar{p}_{1}+\frac{\epsilon}{\sqrt{b}} \cdot \frac{1}{\sqrt{a b} L} \int_{0}^{\sqrt{a b} L} r(t) \cos (t) d t
$$


where $\sqrt{a b} L$ is the period of the orbit of (A.7). passing through $(1,0)$. To simplify the computation, it is convenient to integrate w.r.t. the variable $\theta$.

$$
\tilde{p}_{1}(\epsilon)=\bar{p}_{1}+\frac{\epsilon}{\sqrt{b}} \cdot \frac{1}{\sqrt{a b} L} \int_{0}^{2 \pi} r(\theta) \cos (\theta) \frac{d t}{d \theta} d \theta .
$$

Using (A.3), we get

$$
\tilde{p}_{1}(\epsilon)=\bar{p}_{1}+\frac{\epsilon}{\sqrt{b}} \cdot \frac{1}{\sqrt{a b} L} \int_{0}^{2 \pi} \frac{r(\theta) \cos (\theta)}{1-\epsilon r(\theta) \beta(\theta)} d \theta .
$$

Expanding in powers of $\epsilon$ up to second order, and recalling that $r(\theta)=1+\epsilon r_{1}(\theta)+\mathcal{O}\left(\epsilon^{2}\right)$, we get

$\tilde{p}_{1}(\epsilon)=\bar{p}_{1}+\frac{\epsilon}{\sqrt{b}} \cdot \frac{1}{\sqrt{a b} L} \int_{0}^{2 \pi}\left[1+\epsilon \cdot\left(r_{1}(\theta)+\beta(\theta)\right)+\mathcal{O}\left(\epsilon^{2}\right)\right] \cos (\theta) d \theta=\bar{p}_{1}+\epsilon^{2} \frac{1}{4 a b} \frac{2 \pi}{\sqrt{a b} L}+\mathcal{O}\left(\epsilon^{3}\right)$.

Observing that (A.8) is equivalent to

$$
\frac{2 \pi}{\sqrt{a b} L}=1+\epsilon^{2} \cdot \frac{a+b}{48 a^{2} b^{2}}+\mathcal{O}\left(\epsilon^{3}\right)
$$

we conclude

$$
\tilde{p}_{1}(\epsilon)=\bar{p}_{1}+\epsilon^{2} \frac{1}{4 a b}+\mathcal{O}\left(\epsilon^{3}\right)
$$

An entirely similar computation yields

$$
\tilde{p}_{2}(\epsilon)=\bar{p}_{2}-\epsilon^{2} \frac{1}{4 a b}+\mathcal{O}\left(\epsilon^{3}\right) .
$$

A consequence of the above computations is the following. Call $\widetilde{L}(\epsilon)$ the period of the closed orbits of the linear system

$$
\left\{\begin{array}{l}
z_{1}^{\prime}=-\tilde{p}_{1}(\epsilon) z_{2} \\
z_{2}^{\prime}=-\tilde{p}_{2}(\epsilon) z_{1}
\end{array}\right.
$$

Using (A.9)-(A.10) one obtains

$$
\begin{aligned}
\tilde{L}(\epsilon) & =\frac{2 \pi}{\sqrt{-\tilde{p}_{1}(\epsilon) \tilde{p}_{2}(\epsilon)}} \\
& =\frac{2 \pi}{\sqrt{\left(a+\epsilon^{2} /(4 a b)\right)\left(b+\epsilon^{2} /(4 a b)\right)}}+\mathcal{O}\left(\epsilon^{3}\right) \\
& =\frac{2 \pi}{\sqrt{a b}}\left(1-\epsilon^{2}(a+b) /\left(8 a^{2} b^{2}\right)\right)+\mathcal{O}\left(\epsilon^{3}\right) .
\end{aligned}
$$

Comparing the constants in front of the second order expansion terms of $L(\epsilon)$ and $\tilde{L}(\epsilon)$, for every $\epsilon>0$ small enough one finds

$$
\tilde{L}(\epsilon)<L(\epsilon) .
$$




\section{A.3 A special trajectory.}

Proposition 1 shows the instability of all small periodic travelling waves of (4.8) bifurcating from a steady state solution. Notice that this instability occurs w.r.t. periodic perturbations having twice (or more generally: $N$ times) the basic period. On the other hand, if we analyze the flow of the parabolic system (4.8) within the space of functions having exactly the same period $L(\epsilon)$ as one of the small periodic orbits, an interesting feature appears.

Looking at (4.15) in the case $L=\widetilde{L}$, we see that $B_{0}$ has a double zero eigenvalue, while $B_{1}$ and $B_{-1}$ both have an eigenvalue with real part close to zero, when $L \approx 2 \pi / \sqrt{\left|\tilde{p}_{1} \tilde{p}_{2}\right|}$. All other eigenvalues of the matrices $B_{k}$ have strictly negative real parts.

For a fixed $\epsilon>0$ sufficiently small, by Theorem 6.1.2 in [He], there exists a local invariant manifold $\mathcal{M}$ of dimension 4 containing the periodic solution

$$
p(t, x)=p^{(\epsilon)}(x-\sigma(\epsilon) t)
$$

as well as the constant solution

$$
p(t, x) \equiv \tilde{p}(\epsilon) .
$$

Here $\tilde{p}(\epsilon)$ is the average value of the orbit $p^{(\epsilon)}$, see (A.9)-(A.10). We now observe that the vector valued integral

$$
\int_{0}^{L} p(t, x) d x
$$

remains constant in time along every solution of (4.8). We can thus consider the 2dimensional invariant sub-manifold $\mathcal{M}^{*} \subset \mathcal{M}$ containing all orbits in $\mathcal{M}$ having the same average value $\tilde{p}(\epsilon)$. Of course, this manifold will contain the two above solutions. By the inequality (A.12), the constant solution (A.14) is unstable within $\mathcal{M}^{*}$. Since $\mathcal{M}^{*}$ contains the periodic orbit (A.13) but no other steady state, by the PoincareBendixon theory (see $[\mathrm{HS}]$ ) we conclude that there exists a solution $t \mapsto p(t) \in \mathcal{M}^{*}$ which approaches the unstable steady state $\tilde{p}$ as $t \rightarrow-\infty$, and tends to a periodic orbit for $t \rightarrow \infty$.

\section{References}

[A] J. P. Aubin, Mathematical Methods of Game and Economic Theory, North Holland, 1979.

[BC] M. Bardi and I. Capuzzo Dolcetta, Optimal Control and Viscosity Solutions of Hamilton-Jacobi-Bellman Equations, Birkhäuser, 1997.

[BO] T. Basar and G. J. Olsder, Dynamic Noncooperative Game Theory, $2^{d}$ Edition, Academic Press, London 1995. 
[BB] S. Bianchini and A. Bressan, Vanishing viscosity solutions of nonlinear hyperbolic systems, Annals of Mathematics, to appear.

[BS] A. Bressan and W. Shen, Small BV solution of hyperbolic non-cooperative differential games, SIAM J. Control Optim., to appear.

[CP] P. Cardaliaguet and S. Plaskacz, Existence and uniqueness of a Nash equilibrium feedback for a simple non-zero-sum differential game. Int. J. Game Theory 32 (2003), 33-71.

[FL] H. Frid and I. S. Liu, Oscillation waves in Riemann problems inside elliptic regions for conservation laws of mixed type, Z. Angew. Math. Phys. 46 (1995), 913-931.

[F1] A. Friedman, Differential Games, Wiley-Interscience, 1971.

[F2] A. Friedman, Stochastic Differential Games, J. Differential Equat. 11 (1972), 79108.

[He] D. Henry, Geometric theory of Semilinear Parabolic Equations, Springer Lecture Notes in Math. 840, 1981.

[HS] M. W. Hirsch and S. Smale, Differential Equations, Dynamical Systems and Linear Algebra, Academic Press, New York 1974.

[KY] H. O. Kreiss and J. Yström, Parabolic problems which are ill-posed in the zero dissipation limit, Math. Comput. Modeling 35 (2002), 1271-1295.

[Lx] P. Lax, Asymptotic solution of oscillatory initial value problems, Duke Math. J. 24 (1957), 627-646.

[L] T. P. Liu, Admissible solutions of hyperbolic conservation laws, Amer. Math. Soc. Memoir 240 (1981).

[Mn] P. Mannucci, Nonzero sum stochastic differential games with dicontinuous feedback, SIAM J. Control Optim., to appear.

[Na] J. Nash, Non-cooperative games, Ann. of Math. 2 (1951), 286-295.

[OZ1] M. Oh and K. Zumbrun, Stability of periodic solutions of conservation laws with viscosity: Analysis of the Evans function, Arch. Rational Mech. Anal. 166 (2003), 99-166.

[OZ2] M. Oh and K. Zumbrun, Stability of periodic solutions of conservation laws with viscosity: Pointwise bounds on the Green function, Arch. Rational Mech. Anal. 166 (2003), 167-196.

[O] G. J. Olsder, On open- and closed-loop bang-bang control in nonzero-sum differential games, SIAM J. Control Optim. 40 (2001), 1087-1106.

[P] L. Perko, Differential Equations and Dynamical Systems, Springer-Verlag, 1991. 
[S1] D. Serre, Systems of Conservation Laws I, II. Cambridge University Press, 2000.

[S2] D. Serre, The stability of periodic solutions of viscous conservation laws: Large wavelength perturbations, Preprint

[Sm] S. Smale, The prisoner's dilemma and dynamical systems associated to noncooperative games, in The Collected Papers of Stephen Smale, F. Cucker and R. Wong Eds., World Scientific, 2000.

[VZ] E. M. Vaisbord and V. I. Zhukovskii, Introduction to Multi-players Differential Games and their Applications, Gordon and Breach Science Publishers, 1988. 